Thate: Ueber die Einwirk. v. Reductionsmitteln etc. 145

\title{
Ueber die Einwirkung von Reductionsmitteln auf 0-Nitrophenoxylessigsänre;
}

von

\section{Alexander Thate.}

Das Verhalten der O-Nitrophenoxylessigsäure ${ }^{1}$ ) gegen reducirend wirkende Substanzen wurde zuerst von Fritzs che ${ }^{2}$ ) untersucht. Derselbe wandte in verdünnter Salzsäure gelöstes Zinnchlorür als Reductionsmittel an und erhielt durch dessen Einwirkung auf O-Nitrophenoxylessigsäure eine Substanz, welche er als O-Amidophenoxylessigsäureanhydrid ansprach. Da die O-Nitrophenylessigsäure ein entsprechendes Verhalten zeigt und ebenfalls ein sog. "Anhydrid", das Oxindol, liefert, so wurde eine Reihe von Versuchen zu dem Zwecke angestellt, zu prüfen, ob sich vom O-Amidophenoxylessigsäureanhydrid Fritzsche's ausgehend, Körper darstellen lassen, welche den Derivaten der O-Amidophenylessigsäure entsprechen.

Im Laufe der Untersuchung stellte sich heraus, dass das nach Fritzsche's Vorschrift dargestellte Reductionsprodukt der O-Nitrophenoxylessigsäure niemals reines O-Amidophenoxylessigsäureanhydrid ist, sondern immer einen mehr oder weniger grossen Chlorgehalt aufweist, von welchem es durch Umkrystallisiren nicht befreit werden kann. Aus diesem Grunde wurde zunächst das Verhalten der O-Nitrophenoxylessigsäure gegen reducirend wirkende Substanzen überhaupt in Untersuchung gezogen, und sollen die hierbei erhaltenen Resultate im Folgenden beschrieben werden, während über

1) An Stelle der Bezeichnung O-Nitrooxyphenylessigsäure ist hier und im Folgenden der Namen O-Nitrophenoxylessigsäure gewählt worden. Vergl. Kolbe-von Meyer, Lehrbuch d. organ. Chemie 2, 481, Anmerk.

2) Dies. Journ. [2] 20, 288.

Journal f. prakt. Chemie [2] Bd. 29. 
146 Thate: Ueber die Einwirk. von Reductionsmitteln eine weitere Untersuchung der Derivate des O-Amidophenoxylessigsäureanhydrids, welche gegenwärtig schon in Angriff genommen ist, später berichtet werden soll.

$$
\begin{aligned}
& \text { O-Nitrophenoxylessigsäure, } \\
& \mathrm{C}\left\{\begin{array}{c}
\mathrm{OC}_{6} \mathrm{H}_{4}, \mathrm{NO}_{2} \\
\mathrm{H}_{2}
\end{array}\right\} \mathrm{OOOH}=\mathrm{C}_{8} \mathrm{H}_{7} \mathrm{NO}_{5} \text {. }
\end{aligned}
$$

Zur Darstellung der O-Nitrophenoxylessigsäure wurde anfangs nach der von Fritzsche ${ }^{1}$ ) angegebenen Methode verfahren, welche darin besteht, dass Lösungen von O-Nitrophenolnatrium und monochloressigsaurem Natron gemischt und in einer eisernen Pfanne vorsichtig auf freiem Feuer eingedampft werden. Die Bildung der O-Nitrophenoxylessigsäure erfolgt hierbei nach der Gleichung:

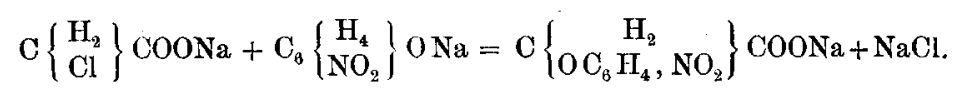

Fritzsche empfiehlt, anfangs ein Molekül Nitrophenolnatrium auf ein Molekül monochloressigsaures Natron zu nehmen, jedoch, sobald die Masse dicker geworden ist, 1 bis $1^{1 / 2}$ Moleküle monochloressigsaures Natron zuzusetzen und die Operation erst dann als beendigt anzusehen, wenn $\mathrm{Zu}$ satz von Natronlauge keine Rothfärbung mehr bewirkt. Folgende, mit möglichster Genauigkeit quantitativ ausgeführte Versuche zeigen, dass die nach diesem Verfahren erzielte

\begin{tabular}{|c|c|c|c|c|c|c|c|c|}
\hline \multicolumn{3}{|c|}{$\begin{array}{l}\text { Nitro- } \\
\text { phenol }\end{array}$} & \multicolumn{3}{|c|}{$\begin{array}{l}\text { Monochlor- } \\
\text { essigsäure }\end{array}$} & \multicolumn{3}{|c|}{$\begin{array}{c}\text { O-Nitrophenoxyl } \\
\text { essigsäure. }\end{array}$} \\
\hline 1) & 50 & Grm. & und & 90 & Grm. & lieferten & $15 \mathrm{C}$ & Grm. \\
\hline 2) & 50 & $"$ & $"$ & 100 & $"$ & $"$ & 19 & . \\
\hline 3) & $\tilde{50}$ & " & $n$ & 80 & " & ", & 28 & $"$ \\
\hline 4) & 30 & $"$ & $n$ & 70 & , & $"$ & 8 & " \\
\hline 5) & 30 & $"$ & " & 80 & $"$ & $n$ & 17,5 & " \\
\hline 6) & 30 & $"$ & $"$ & 80 & $"$ & $"$ & 10,5 & $"$ \\
\hline
\end{tabular}
Ausbeute an O-Nitrophenoxylessigsäure sehr wenig constant ist und deshalb viel zu wünschen übrig lässt.

1) A. a. O. 
Verhältniss der Moleculargewichte der verwendeten Mengen von Nitrophenol und Monochlor-
$(=1)$

1) $1: \quad 2,6$

2) $1 \quad: \quad 2,9$

3) 1 : 2,4

4) $1: 3,4$

5) $1: 3,9$

6)
Ausbeute berechnet auf die verwendeten Mengen von

\begin{tabular}{|c|c|}
\hline $\begin{array}{l}\text { Nitro- } \\
\text { phenol und }\end{array}$ & $\begin{array}{l}\text { Monochlor- } \\
\text { essigsäure }\end{array}$ \\
\hline $21,2 \%$ & $8,0 \%$ \\
\hline 26,8, & 9,1, \\
\hline 39,5, & $16,8 n$ \\
\hline 18,8, & 5,5, \\
\hline 41,1, & 10,5, \\
\hline 24,7, & 6,3 \\
\hline
\end{tabular}

Bei weiteren zehn Versuchen schwankte die Ausbeute an O-Nitrophenoxylessigsäure auf die verwendete Menge O-Nitrophenol berechnet: zwischen 16,2\%-39,4\%, im Mittel $27,1 \%$, und auf die dazu verbrauchte Monochloressigsäure bezogen: zwischen 4,2\%-19,2\%, im Mittel 10,7\%.

Wie die weiter unten angegebenen Versuche zeigen, rühren diese schlechten Ausbeuten daher, dass während des Eindampfens immer ein beträchtlicher Theil Nitrophenol frei wird und sich verflüchtigt. Möglicherweise giebt die entstehende freie O-Nitrophenoxylessigsäure unter gleichzeitiger Bildung von neutralem glycolsaurem Natron die Veranlassung hierzu, wenigstens kann man beobachten, dass die ursprünglich neutrale Reaction der Flüssigkeit je nach jhrer Concentration beim Eindampfen über freiem Feuer bald schneller, bald langsamer verschwindet, und saure Reaction eintritt. Versucht man diese durch Zusatz von Natronlauge abzustumpfen, so liegt die Gefahr nahe, durch einen etwaigen Ueberschuss von Alkali eine weitere Zersetzung von Monochloressigsäure in Glycolsäure zu veranlassen. Einen Ueberschuss von Monochloressigsäure anzuwenden empfiehlt sich nicht; die Versuche zeigen, dass die erzielten Ausbeuten keinen Ersatz dafür gewähren.

Im Ganzen ist die von Fritzsche vorgeschlagene Methode zeitraubend und erfordert grosse Aufmerksamkeit von Seiten des Experimentators. Leicht kann bei ihr durch zu starkes Eindampfen die gebildete O-Nitrophenoxylessigsäure wieder zersetzt werden, ganz abgesehen davon, dass die letztere meist ziemlich unrein ist und zwei- bis dreimal umkrystallisirt werden muss, bevor sie chemisch rein erhalten wird. 


\section{Thate: Ueber die Einwirk. von Reductionsmitteln}

Bedeutend bessere Ausbeute an solcher und beträchtliche Ersparniss an Herstellungsmaterial lässt sich erzielen, wenn in folgender Weise verfahren wird:

Man bringt in einen Kolben ein Gemisch äquivalenter Mengen von $\mathrm{O}$-Nitrophenolnatrium und monochloressigsaurem Natron in möglichst concentrirter Lösung, versieht den Kolben mit einem durchbohrten Kork, fügt ein nicht zu enges Glasrohr ein und stellt den so beschickten Apparat in ein bedecktes Wasserbad, welches auf Siedetemperatur erhalten wird. Schon nach kurzer Zeit entsteht Orthonitrophenoxylessigsäure, jedoch erst nach längerem Erhitzen auf $100^{\circ}$ ist die Umsetzung vollendet, was daran erkannt werden kann, dass die ursprünglich schön weinrothe Färbung der Flüssigkeit einer gelben bis rothgelben gewichen ist. Auch hierbei wird ein grosser Theil des angewendeten Nitrophenolnatriums in der Weise zersetzt, dass Nitrophenol frei wird, dasselbe kann jedoch nicht entweichen, sondern bleibt im Kolben oder sublimirt in das aufgesetzte Glasrohr. Nach Beendigung des Processes lässt man erkalten, filtrirt vom ausgeschiedenen Nitrophenol ab und säuert das Filtrat mit Salzsäure an. Die ausfallende O-Nitrophenoxylessigsäure ist nur durch wenig Nitrophenol verunreinigt und wird durch einmaliges Umkrystallisiren aus viel Wasser, welchem man einige Tropfen Salzsäure zusetzt, vollkommen rein erhalten.

Zur Prüfung der Ausbeute, welche die geschilderte Darstellungsmethode an O-Nitrophenoxylessigsäure gewährt, wurden folgende quantitative Versuche ausgeführt:

1) 30 Grm. Nitrophenol and 20 Grm. Monochloressigsäure lieferten nach 20 stündigem Erhitzen $9 \mathrm{Grm}$. reine O-Nitrophenoxylessigsäure, während $20 \mathrm{Grm}$. freies Nitrophenol zurückerhalten wurden.

2) $30 \mathrm{Grm}$. Nitrophenol und $20 \mathrm{Grm}$. Monochloressigsäure lieferten nach 10 stündigem Erhitzen 13 Grm. reine O-Nitrophenoxylessigsäure und $18 \mathrm{Grm}$. freies Nitrophenol

3) $30 \mathrm{Grm}$, Nitrophenol und $20 \mathrm{Grm}$. Monochloressigsäure lieferten nach 11 stündigem Erhitzen 17 Grm. reine O-Nitrophenoxylessigsäure und $11 \mathrm{Grm}$. freies Nitrophenol.

4) 30 Grm. Nitrophenol und 20 Grm. Monochloressig- 
säure lieferten nach 5 stündigem Erhitzen 8 Grm. reine O-Nitrophenoxylessigsäure und $5 \mathrm{Grm}$. freies Nitrophenol, während noch 11 Grm. Nitrophenol in der Flüssigkeit als Nitrophenolnatrium enthalten waren.

1) $20 \mathrm{Stdn}$. mit

Verhältn. d. Moleculargew. Ausbeute berechn. auf die in

Daner der in Reaction getretenen des Mengen von

\section{Erhitzens Nitrophenol Monochlor-} $(=1)$ u. essigsäure Reaction getretenen Mengen von

$$
\begin{aligned}
& \text { Nitro- } \\
& \text { phenol }
\end{aligned} \text { u. } \begin{gathered}
\text { Monochlor- } \\
\text { essigsäure }
\end{gathered}
$$

Unterbrechung $1: 2,9 \quad 63,5 \% \quad 21,6 \%$

2) 10 Stdn. mit

Unterbrechung $1 \quad: \quad 2,4 \quad 76,4, " 31,2$,

3) 11 Stunden

ununterbrochen $1 \quad: \quad 1,6$

4) 5 Stunden

ununterbrochen $1: 2,1$

$\begin{array}{ll}63,2, & 40,8, \\ 40,3, & \quad 19,2,\end{array}$

Aus den gefundenen Werthen ist ersichtlich, dass in der Regel 10-11 stïndiges Erhitzen erforderlich ist, um die möglichst grösste Ausbeute an $\mathrm{O}$-Nitrophenoxylessigsäure zu erzielen. Leider lässt dieselbe, namentlich wenn sie auf die verwendete Menge von Monochloressigsäure bezogen wird, immer noch zu wünschen übrig, doch dürfte sich das angegebene Verfahren vor Allem deswegen empfehlen, weil der zur Verwendung kommende Apparat wenig oder gar keiner Beaufsichtigung bedarf.

Ganz besonders mag noch darauf aufmerksam gemacht werden, dass bei der Darstellung von O-Nitrophenolnatrium und monochloressigsaurem Natron jeder Ueberschuss von freiem oder kohlensaurem Alkali sorgfältig vermieden werden muss; wendet man diese Vorsicht nicht an, so wird die Ausbeute durch - sich bildende Glycolsäure stark beeinträchtigt.

$\mathrm{Zu}$ gleichen Resultaten gelangt man, wenn äquivalente Mengen von O-Nitrophenolnatrium und monochloressigsaurem Natron in wässriger Lösung im Einschmelzrohr auf $100^{\circ}$ erhitzt werden; nur dass in diesem Falle der Process etwas schneller verläuft.

Die in oben angegebener $\mathrm{W}$ eise dargestellte $\mathrm{O}$-Nitrophenoxylessigsäure krystallisirt aus Wasser in unvollkommen 
ausgebildeten Pyramiden, welche sich zu spiessigen Aggregaten vereinigen. In regulären Octaëdern, der Angabe Fritzsche's entsprechend, konnte sie nicht krystallisirt erhalten werden. Zwar zeigten die sie bildenden Pyramiden oft Axenverhältnisse, welche ihre Einreihung in das reguläre System zu rechtfertigen schienen, doch wurde andererseits durch Anwendung von polarisirtem Licht Doppelbrechung der Substanz und somit ihre Zugehörigkeit zu einem anderen als dem regulären Krystallsystem nachgewiesen.

Die Säure schmilzt bei $156,5^{\circ}$, zeigt gelblichweisse Färbung und besitzt im Uebrigen die von Fritzsche ausführlich beschriebenen Eigenschaften. Thre Reinheit bestätigen ausserdem folgende Analysen:

1) $0,3444 \mathrm{Grm}$. Substanz lieferten beim Verbrennen mit Kupferoxyd 0,6155 Grm. $\mathrm{CO}_{2}$ (entsprech. 0,16786 Grm. C) und 0,1170 Grm. $\mathrm{H}_{2} \mathrm{O}$ (entsprech. 0,01303 Grm. H).

2) 0,2453 Grm. Substanz lieferten beim Verbrennen mit Kupferoxyd $15,0 \mathrm{Ccm} . \mathrm{N}$ bei $17^{\circ}$ und $764 \mathrm{Mm}$. Bar. (entsprechend 0,0175 (trm. N).

\begin{tabular}{cccc} 
Die Substanz $\mathrm{C}_{8} \mathrm{H}_{7} \mathrm{NO}_{5}$ & \multicolumn{2}{c}{ Gefunden. } \\
enthält: & 1. & 2. \\
$\mathrm{C}_{8}=96=48,73 \%$ & 48,74 & - \\
$\mathrm{H}_{7}=7=3,55 \%$ & 3,78 & - \\
$\mathrm{N}=14=7,11 \%$ & - & 7,13 \\
$\mathrm{O}_{5}=80=40,61 \%$ & - & - \\
$197 \quad 100,00$ & &
\end{tabular}

Reduction der O-Nitrophenoxylessigsäure.

Als reducirende Mittel, deren Einwirkung auf O-Nitrophenoxylessigsäure theils in wässriger, theils in alkoholischer Lösung geprüft wurde, kamen Natriumamalgam, Schwefelammonium, Eisen mit Essigsäure und Zinnchlorür in salzsaurer Lösung zur Verwendung.

Die Reduction liefert immer dasselbe Endprodukt, nämlich O-Amidophenoxylessigsäure beziehentlich deren „Anhydrid", nur wird dieses bei Anwendung sauer reagirender Lösungen immer direct erhalten, während durch Reduction in alkalisch reagirenden Lösungen verschiedene, von der 
O-Nitrophenoxylessigsäure sich ableitende Azoverbindungen als $\mathbf{Z}$ wischenstufen isolirt werden können.

In der folgenden Beschreibung des Reductionsvorganges wird der grösseren Uebersichtlichkeit wegen von diesem Unterschied Gebrauch gemacht, und Reduction in alkalischer und solche in saurer lösung unterschieden werden.

A. Reduction in alkalisch reagirenden Lösungen.

Das geeignetste Mittel zur Reduction in alkalisch reagirender Lösung ist das Natriumamalgam aus dem Grunde, weil man bei seiner Verwendung in jedem Augenblicke mit ziemlicher Sicherheit angeben kann, bis zu welchem Grade die Reduction vorgeschritten ist. Als Haupterforderniss muss vorausgesetzt werden, dass man ein gleichmässig gemischtes Amalgam von genau bekanntem Gehalt an Alkali - am besten $4 \%$ - zur Verfügung hat und dieses nur in kleinen Portionen in die zu reducirende Lösung einträgt. Sind beide Bedingungen erfüllt, so genügt fast die theoretisch nöthige Menge.

Der durch Zersetzung von Natriumamalgam frei werdende Wasserstoff wirkt auf eine wässrige Lösung von orthonitrophenoxylessigsaurem Natron in der Weise ein, dass der Reihe nach Azoxyorthophenoxylessigsäure, Azoorthophenoxylessigsäure, Hydrazoorthophenoxylessigsäure und Amidoorthophenoxylessigsäure gebildet werden. Der Verlauf der Reduction lässt sich durch folgende Gleichungen veranschaulichen $\left.{ }^{1}\right)$ :

I. $2\left\{\mathrm{C}\left\{\begin{array}{l}\mathrm{H}_{2} \\ \mathrm{OC}_{6} \mathrm{H}_{4}, \mathrm{NO}_{2}\end{array}\right\} \mathrm{COOH}\right\}+3 \mathrm{H}_{2}=\left\{\begin{array}{l}\mathrm{C}\left\{\begin{array}{l}\mathrm{H}_{2}, \mathrm{COOH} \\ \mathrm{OC}_{6} \mathrm{H}_{4}, \mathrm{~N}\end{array}\right. \\ \mathrm{C}\left\{\begin{array}{l}\mathrm{OC}_{6} \mathrm{H}_{4}, \mathrm{~N} \\ \mathrm{H}_{2}, \mathrm{COOH}\end{array}\right\}\end{array}\right\}+3 \mathrm{H}_{2} \mathrm{O}$.

1) Um Wiederholungen zu vermeiden, wurden an dieser Stelle die chemischen Vorgänge, welche die angeführten Gleichungen veranschaulichen sollen, nicht in Worten wiedergegeben. Die erforderlichen Erklärungen finden sich bei der Beschreibung der einzelnen Verbindungen. - Der Stickstoff ist in den hier für die Azoverbindungen gebrauchten Formeln immer als dreiwerthig angenommen worden. 
152 Thate: Ueber die Einwirk. von Reductionsmitteln

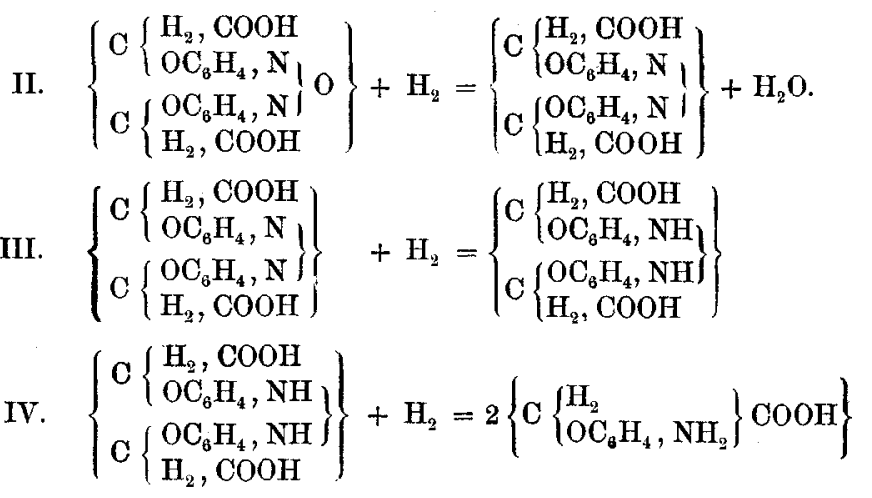

Einigermaassen erschwert wird die Reingewinnung der genannten Azoverbindungen dadurch, dass die durch die Gleichungen I-IV dargestellten Vorgänge nicht streng nach einander, sondern zum Theil neben einander verlaufen. Am leichtesten wird Azoorthophenoxylessigsäure erhalten, am schwierigsten dagegen Hydrazoorthophenoxylessigsäure. Zum grössten Theil scheint dieser Uebelstand seine Begründung darin zu haben, dass die zu reducirende Lösung infolge ihres während des Processes wachsenden specifischen Gewichtes nicht immer gleichmässig gemischt ist. Häufiges Umrühren oder noch besser Umschütteln ist deshalb unerlässlich. Nähere Angaben über das Verfahren finden sich bei der Beschreibung der Darstellungsweisen der einzelnen Verbindungen.

1. Azoxyorthophenoxylessigsäure,

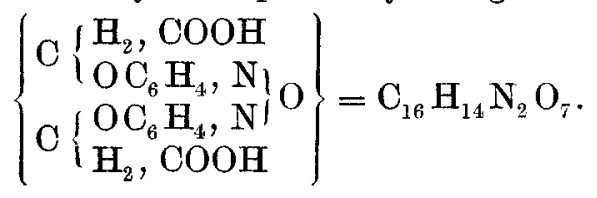

Azoxyorthophenoxylessigsäure bildet sich, wenn den beiden Nitrylen, welche in je zwei Molekülen O-Nitrophenoxylessigsäure enthalten sind, durch sechs Atome nascirenden Wasserstoffs drei A tome Sauerstoff unter gleichzeitiger Bildung von drei Molekülen Wassèr entzogen werden. Dieser Vorgang wird durch die Gleichung $I$ auf Seite 151 ausgedrückt. 
Genannte Säure entsteht ferner durch Oxydation von Hydrazoorthophenoxylessigsäure.

Am zweckmässigsten stellt man sie auf folgende Weise dar:

Eine warme Lösung von 5 Thln. trocknen kohlensauren Natrons in $140 \mathrm{Thln}$. Wasser wird durch Hinzufügen von 18,6 Thln. O-Nitrophenoxylessigsäure genau neutralisirt. In die so erhaltene, etwa 15 proc. Lösung von o-nitrophenoxylessigsaurem Natron werden 160-170 Thle. 4proc. Amalgams nach und nach in kleinen Portionen unter häufigem Umschütteln eingetragen. Hierbei tritt keine sichtbare Entbindung von Wasserstoff, dagegen ziemlich starke Erwärmung ein. Man hat dafür zu sorgen, dass die Temperatur der Lösung möglichst zwischen $50^{\circ}-60^{\circ}$ erhalten wird. Die ursprünglich weingelbe Masse färbt sich im Verlaufe der Reduction roth. Nachdem alles Natriumamalgam eingetragen ist, wird die Flüssigkeit vom ausgeschiedenen Quecksilber abgegossen und erkalten gelassen. Sie erstarrt hierbei zu einem gelben Brei, welchen man absaugt und in wenig Wasser löst. Die Lösung ist, nachdem sie filtrirt wurde, mit Salzsäure anzusäuern, die ausgeschiedene Substanz abzusaugen, mit Wasser zu waschen und aus stark verdünntem Alkohol umzukrystallisiren. Der so erhaltene, lange gelbe Nadeln bildende Körper ist noch keine reine Azoxyorthophenoxylessigsäure, sondern ein Gemisch von letzterer und Azoorthophenoxylessigsäure. Um ein vorläufiges Urtheil über den Verlauf der Reduction fällen zu können, nimmt man eine Schmelzpunktbestimmnng der Substanz vor. Schmilzt dieselbe bei $162^{\circ}$ oder niedriger, so würde es unzweckmässig sein, sie weiter auf Azoxyorthophenoxylessigsäure zu verarbeiten, da in diesem Falle die Reduction weiter fortgeschritten ist, und hauptsächlich Azoorthophenoxylessigsäure sich gebildet hat. Ist der Versuch nach Wunsch verlaufen, so muss der gefundene Schmelzpunkt bei $170^{\circ}$ oder darüber liegen. Zur Reindarstellung der Azoxyorthophenoxylessigsäure wird das gewonnene Säuregemisch ätherificirt. Man löst es zu diesem Zwecke in starkem Alkohol und leitet trocknes Salzsäuregas bis zur Sättigung, anfangs ohne Er- 


\section{Thate: Ueber die Einwirk. von Reductionsmitteln}

wärmen, später unter Erhitzen im Wasserbade, ein. Bei genügender Concentration erstarrt nach Beendigung des Processes die ganze Masse beim Erkalten zu einem rothen Krystallbrei; derselbe ist abzusaugen und mit verdünntem Alkohol zu waschen. Der Alkohol löst den gleichzeitig entstandenen Aethyläther der Azoorthophenoxylessigsäure, welcher die intensive Rothfärbung verursacht, so dass die Substanz sich während des Auswaschens hellgelb färbt. Durch mehrmaliges Umkrystallisiren aus Alkohol und Behandeln mit Thierkohle wird der auf dem Filter zurückbleibende Aethyläther der Azoxyorthophenoxylessigsäure in langen, weissen Nadeln erhalten. Um aus dem azoxyorthophenoxylessigsauren Aethyl die freie Azoxyorthophenoxylessigsäure darzustellen, führt man es durch Zersetzen mit Kalilauge in das Kalisalz derselben über, zerlegt letzteres durch Salzsäure und krystallisirt die ausfallende Azoxyorthophenoxylessigsäure aus Wasser um.

Die beschriebene Darstellungsmethode gewährt wechselnde Ausbeuten von der gewünschten Säure, weil durch das mehrfach zu wiederholende Umkrystallisiren ein Verlust nicht zu vermeiden ist oder nur auf Kosten der Reinheit der Substanz zu umgehen sein würde.

Die mit der mehrmals umkrystallisirten Säure vorgenommenen analytischen Bestimmungen lieferten folgende Resultate:

1) 0,1890 Grm. bei $120^{\circ}-130^{\circ}$ getrockneter Substanz lieferten beim Verbrennen mit Kupferoxyd $13,4 \mathrm{Ccm}$. $\mathrm{N}$ bei $12^{\circ}$ und $746 \mathrm{Mm}$. Bar. (entsprechend 0,0156 Grm. N).

2) $0,1780 \mathrm{Grm}$. bei $120^{\circ}-130^{\circ}$ getrockneter Substanz lieferten beim Verbrennen mit Kupferoxyd $0,3620 \mathrm{Grm} . \mathrm{CO}_{2}$ (entspr. 0,09873 Grm. C) und 0,0680 Grm. H,O (entspr, 0,00755 Grm. H).

3) $0,2335 \mathrm{Grm}$. bei $120^{\circ}-130^{\circ}$ getrockneter Substanz lieferten beim Verbrennen mit Kupferoxyd 0,4740 Grm. $\mathrm{CO}_{2}$ (entspr. 0,12927 Grm. C) und 0,0905 Grm. $\mathrm{H}_{2} \mathrm{O}$ (entspr. 0,01005 Grm. H).

4) 0,2880 Grm. aus Wasser umkrystallisirte, lufttrockne Substanz verloren beim Trocknen bei $125^{\circ}$ bis zum constanten Gewicht 0,0143 Grm. $\mathrm{H}_{2} \mathrm{O}$.

5) 0,1640 Grm. aus Wasser umkryst., lufttrockne Subst. verloren beim Trocknen bei $125^{\circ}$ bis zum constanten Gew. 0,0080 Grm. $\mathrm{H}_{2} \mathrm{O}$.

6) 0,1125 Grm. aus Wasser umkrystallisirte, lufttrockne Substanz, welche 5-6 Tage mit der Mutterlauge in Berührung gewesen waren, 
verloren beim Trocknen bei $130^{\circ}$ bis zum constanten Gewicht 0,0080 Grm. $\mathrm{H}_{2} \mathrm{O}$.

$$
\begin{aligned}
& \text { Die Substanz } \mathrm{C}_{16} \mathrm{H}_{14} \mathrm{~N}_{2} \mathrm{O}_{7} \\
& \text { enthält: } \\
& \mathrm{C}_{16}=192=55,49 \% \\
& \mathrm{H}_{14}=14=4,05 \text { " } \\
& \mathrm{N}_{2}=28=8,09 \text {, } \\
& \mathrm{O}_{7} \frac{=112=32,37, "}{346 \quad 100,00}
\end{aligned}
$$

Die Substanz $\mathrm{C}_{16} \mathrm{H}_{14} \mathrm{~N}_{2} \mathrm{O}_{7}+\mathrm{H}_{2} \mathrm{O}$ enthält:

$$
\begin{aligned}
& \mathrm{C}_{16} \mathrm{H}_{14} \mathrm{~N}_{2} \mathrm{O}_{7}=346=95,05 \% \\
& \mathrm{H}_{2} \mathrm{O}=\frac{18=4,95}{364}=\frac{100,00}{2}
\end{aligned}
$$

Die Substanz $\mathrm{C}_{16} \mathrm{H}_{14} \mathrm{~N}_{2} \mathrm{O}_{7}+1 / 2 \mathrm{H}_{2} \mathrm{O}$ enthält:

$$
\begin{aligned}
& \mathrm{C}_{16} \mathrm{H}_{14} \mathrm{~N}_{9} \mathrm{O}_{7}=346=92,76 \% \\
& 11 / 2 \mathrm{H}_{2} \mathrm{O}=27=7,24 \% \\
& \cline { 2 - 3 }=
\end{aligned}
$$

Gefunden:

\begin{tabular}{lcr}
\hline 1. & 2. & 3. \\
- & 55,46 & 55,36 \\
- & 4,24 & 4,31 \\
8,25 & - & - \\
- & - & -
\end{tabular}

Gefunden:

4.

5.

$\overline{4,97} \quad \overline{4,88}$

Gefunden:

6.

7,11

Die Azoxyorthophenoxylessigsäure krystallisirt aus wässerigen oder verdünnt alkoholischen Lösungen mit einem Molekül Krystallwasser entweder in kurzen, schlecht ausgebildeten Prismen oder in Skalenoëdern. Die letzteren Krystallformen zeigen grosse Aehnlichkeit mit den entsprechenden des Kalkspaths. Sie stimmen auch darin mit jenen überein, dass, sie häufig von zahlreichen Blätterdurchgängen beziehentlich Spaltungsflächen durchsetzt sind und rhomboëdrisch spalten. Lässt man die Krystalle längere Zeit mit ihrer Mutterlauge in Berührung, so gehen sie immer freiwillig in Rhomboëder über, indem sie noch ein halbes Molekül Krystallwasser aufnehmen.

Die wasserfreie Substanz sieht fast rein weiss, die ein Molekül Krystallwasser besitzende schwefelgelb aus, während die Farbe der mit $1 \frac{1}{2}$ Mol. Wasser auskrystallisirten Rhomboëder kanariengelb ist. Um den Körper wasserfrei zu erhalten, ist längeres Erhitzen auf $120^{\circ}-130^{\circ}$ erforderlich. Ausser in Wasser und Alkohol, in 'denen Azoxyorthophenoxylessigsäure mit hellgelber Farbe löslich ist, wird sie mit derselben Färbung noch gelöst von wässrigen Alka- 
156 Thate: Ueber die Einwirk. von Reductionsmitteln

lien, Salzsäure und Eisessig. Dagegen löst sie sich in starker Salpetersäure und concentrirter Schwefelsäure in der Kälte mit rother Farbe.

In wasserfreiem Aether und Benzol ist sie unlöslich. Der Schmelzpunkt der Säure liegt bei $186^{\circ}-187^{\circ}$. Erhitzt man sie vorsichtig auf einem Platinblech über der Flamme, so schmilzt sie zunächst zu einer gelben Flüssigkeit, verpufft stärker erhitzt unter Ausstossen gelber Dämpfe und Zurücklassen einer voluminösen, schwer verbrennlichen Kohle. Eine warme wässrige Lösung von Azoxyorthophenoxylessigsäure reagirt stark sauer, giebt mit essigsaurem Blei versetzt sofort einen gelblich weissen, flockigen Niederschlag, während salpetersaures Silber und Chlorbaryum in der Wärme keinen solchen hervorbringen und erst beim Erkalten der Lösung hellgelb gefärbte, krystallinische Niederschläge verursachen. Giesst man zu einer Lösung der Säure in absolutem Alkohol alkoholisches Ammoniak, so erfolgt sofort Ausscheidung von gelbem azoxyorthophenoxylessigsaurem Ammon.

Im Allgemeinen ist die neue Säure als solche gut charakterisirt; sie vermag Kohlensäure aus ihren Verbindungen in Freiheit zu setzen und liefert schön krystallisirende Salze. Bei der Darstellung der letzteren geht man am vortheilhaftesten rom Ammonsalz aus.

Azoxyorthophenoxylessigsaures Ammon,

$$
\left\{\begin{array}{c}
\mathrm{C}\left\{\begin{array}{l}
\mathrm{H}_{2}, \mathrm{COONH}_{4} \\
\mathrm{OC}_{8} \mathrm{H}_{4}, \mathrm{~N} \\
\mathrm{C}
\end{array}\right\} \begin{array}{l}
\mathrm{OC}_{6} \mathrm{H}_{4}, \mathrm{~N} \\
\mathrm{H}_{2}, \mathrm{COONH}
\end{array}
\end{array}\right\}=\mathrm{C}_{16} \mathrm{H}_{20} \mathrm{~N}_{4} \mathrm{O}_{7} .
$$

Zur Darstellung von azoxyorthophenoxylessigsaurem Ammon löst man reine Azoxyorthophenoxylessigsäure in $991 / 2 \%$ Alkohol und versetzt die Lösung entweder mit starkem alkoholischem Ammoniak oder leitet in dieselbe einen Strom trocknen Ammoniakgases ein. In beiden Fällen fällt das chemisch reine Salz als mikrokrystallinischer Niederschlag nieder, welcher aus hellgelb gefärbten, schlecht conturirten Blättchen besteht. 
Analyse:

$0,1535 \mathrm{Grm}$. über Schwefelsäure getrockneter Substanz lieferten beim Verbrennen mit Kupferoxyd $19,1 \mathrm{Ccm} . \mathrm{N}$ bei $11^{\circ}$ und $748 \mathrm{Mm}$. Bar. (entspr. 0,022412 Grm. N).

Die Substanz $\mathrm{C}_{16} \mathrm{H}_{20} \mathrm{~N}_{4} \mathrm{O}_{\tau}$ enthält: Gefunden:

$$
\mathrm{N}=14,74 \% \quad 14,60
$$

Azoxyorthophenoxylessigsaures Ammon ist in Wasser und verdünntem Alkohol leicht löslich, in absolutem Alkohol und Aether dagegen unlöslich.

Die wässrige Lösung des Salzes besitzt weingelbe Färbung, reagirt neutral und giebt folgende Reactionen:

$\mathrm{BaCl}_{2}$ : gelblich weisser, krystallinischer Niederschlag, im Ueberschuss des Fällungsmittels auch beim Erwärmen nicht löslich, in Essigsäure löslich. $\mathrm{Pb}\left(\mathrm{C}_{2} \mathrm{H}_{3} \mathrm{O}_{2}\right)_{2}$ : gelblich weisser Niederschlag, im Ueberschuss des Fällungsmittels beim Erwärmen löslich, in Essigsäure ebenfalls löslich.

$\mathrm{AgNO}_{3}$ : hellgelber Niederschlag, im Ueberschuss des Fällungsmittels beim Erwärmen nur wenig löslich, in Essigsäure und Salpetersäure löslich.

$\mathrm{Fe}_{2} \mathrm{Cl}_{6}$ : hellgelber Niederschlag, im Ueberschuss des Fällungsmittels beim Erwärmen etwas löslich, in Salzsäure löslich.

$\mathrm{CuSO}_{4}$ : hellgrüner Niederschlag.

$\mathrm{Hg} \mathrm{Cl}_{2}$ und $\mathrm{MgSO}_{4}$ liefern keinen Niederschlag.

Azoxyorthophenoxylessigsaures Silber (neutrales Salz),

$$
\left\{\begin{array}{l}
\mathrm{C}\left\{\begin{array}{l}
\mathrm{H}_{3}, \mathrm{COOAg} \\
\mathrm{OC}_{6} \mathrm{H}_{4}, \mathrm{~N} \\
\mathrm{CO}_{6} \mathrm{H}_{4}, \mathrm{~N}
\end{array}\right\} \mathrm{O} \\
\mathrm{H}_{2}, \mathrm{COOAg}
\end{array}\right\}=\mathrm{C}_{16} \mathrm{H}_{12} \mathrm{~N}_{2} \mathrm{O}_{7} \mathrm{Ag}_{2} .
$$

Wird eine wässrige Lösung von neutralem azoxyorthophenoxylessigsaurem Ammon mit salpetersaurem Silber versetzt, so fällt das neutrale Silbersalz als hellgelber Niederschlag aus. Man saugt denselben $a b$, wäscht mit Wasser und krystallisirt aus Wasser um. 
158 Thate: Ueber die Einwirk. von Reductionsmitteln

\section{Analysen:}

1) $0,1385 \mathrm{Grm}$. bei $100^{\circ}-110^{\circ}$ getroekneter Substanz lieferten beim Einäsehern 0,0530 Grm. Silber.

2) $0,1210 \mathrm{Grm}$. bei $100^{\circ}-110^{\circ}$ getrockneter Substanz lieferten 0,0465 Grm. Silber.

$$
\begin{array}{rlcc}
\text { Die Substanz } \mathrm{C}_{16} \mathrm{H}_{12} & \mathrm{~N}_{2} \mathrm{O}_{7} \mathrm{Ag}_{2} & \multicolumn{2}{c}{\text { Gefunden: }} \\
\text { enthält: } & \multicolumn{1}{c}{\text {. }} & 2 . \\
\mathrm{C}_{16} \mathrm{H}_{12} \mathrm{~N}_{2} \mathrm{O}_{7} & =344=61,43 \% & - & - \\
\mathrm{Ag}_{2} & =216=38,57, & 38,27 & 38,43 \\
& =260 \quad 100,00 & &
\end{array}
$$

Das Salz ist in Wasser and Alkohol mit hellgelber Farbe löslich, es krystallisirt aus ersterem Lösungsmittel wasserfrei in kleinen, gut ausgebildeten, monoklinen Krystallen, deren Habitus grosse Aehnlichkeit mit demjenigen des Orthoklases besitzt. Die Farbe der krystallisirten Substanz ist kanariengelb. Sie zersetzt sich beim Erhitzen auf $130^{\circ}-140^{\circ}$ allmählich unter Bräunung; einer höheren Temperatur ausgesetzt erfolgt die Zersetzung plötzlich unter Freiwerden gelber Dämpfe. Aus diesem Grunde ist die directe Bestimmung ihres Silbergehaltes durch Einäschern häufig mit einem Verlust verbunden. Umgehen lässt sich ein solcher, wenn die Analyse in der Weise vorgenommen wird, dass man die Substanz in einem Porcellantiegel mit wenig Alkohol übergiesst und diesen anzündet.

Azoxyorthophenoxylessigsaures Silber

$$
\left\{\begin{array}{l}
\text { (saures Salz), } \\
\left.\qquad \begin{array}{l}
\mathrm{H}_{2}, \mathrm{COOAg} \\
\mathrm{OC}_{6} \mathrm{H}_{4}, \mathrm{~N} \\
\mathrm{C}
\end{array}\right\} \mathrm{O} \mathrm{OC}_{6} \mathrm{H}_{4}, \mathrm{~N} \\
\mathrm{H}_{2}, \mathrm{COOH}
\end{array}\right\}=\mathrm{C}_{16} \mathrm{H}_{13} \mathrm{~N}_{2} \mathrm{O}_{7} \mathrm{Ag} .
$$

Saures azoxyorthophenoxylessigsaures Silber wurde dadurch erhalten, dass eine warme wässrige Lösung von Azooxyorthophenoxylessigsäure mit salpetersaurem Silber versetzt, der sich beim Erkalten bildende Niederschlag von neutralem und saurem Silbersalz abfiltrirt, und das Filtrat weiter eingeengt worden war. Nach längerem Stehen des letzteren schieden sich aus ihm schwefelgelbe Krystalle ab, 
welche aus Wasser umkrystallisirt und über Chlorcalcium getrocknet wurden.

Analyse:

0,1485 Grm. Substanz lieferten beim Einäschern 0,0850 Grm. Silber.

Der Körper $\mathrm{C}_{16} \mathrm{H}_{13} \mathrm{~N}_{2} \mathrm{O}_{7} \mathrm{Ag}$ enthält: Gefunden:

$$
\begin{array}{rlr}
\mathrm{C}_{16} \mathrm{H}_{18} \mathrm{~N}_{2} \mathrm{O}_{7} & =345=76,16 \% \\
\mathrm{Ag} & =108=23,84 \% & - \\
& &
\end{array}
$$

Das saure Silbersalz ist leichter löslich als das neutrale; seine wässrige Lösung reagirt schwach sauer.

Azoxyorthophenoxylessigsaurer Baryt, $\left\{\begin{array}{l}\mathrm{C}\left\{\begin{array}{l}\mathrm{H}_{2}, \mathrm{COO} \\ \mathrm{OC}_{6} \mathrm{H}_{4}, \mathrm{~N} \\ \mathrm{O}\left\{\begin{array}{l}\mathrm{OC}_{6} \mathrm{H}_{4}, \mathrm{~N} \\ \mathrm{H}_{2}, \mathrm{COO}\end{array}\right.\end{array}\right\} \mathrm{O}\end{array}\right\}+2 \mathrm{H}_{2} \mathrm{O}=\mathrm{C}_{16} \mathrm{H}_{12} \mathrm{~N}_{2} \mathrm{O}_{7} \mathrm{Ba}+2 \mathrm{H}_{2} \mathrm{O}$.

Beim Versetzen einer wässrigen Lösung von azoxyorthophenoxylessigsaurem Ammon mit Chlorbaryum fällt das Barytsalz als hellgelber, schwerer, krystallinischer Niederschlag aus. Man hat, um den Körper rein zu erhalten, nur nöthig, ihn auf einem Filter zu sammeln und mehrere Male mit Wasser auszuwaschen.

\section{Analysen:}

1) 0,1315 Grm. lufttrockner Substanz lieferten beim Einäschern $0,0495 \mathrm{Grm} . \mathrm{Ba} \mathrm{CO}_{3}$ (entsprech. 0,034424 Grm. Ba).

2) $0,1190 \mathrm{Grm}$. lufttrockner Substanz verloren beim Trocknen bei $120^{\circ}$ bis zum constanten Gewichte $0,0085 \mathrm{Grm} . \mathrm{H}_{2} \mathrm{O}$ und lieferten beim Einäschern 0,0450 Grm. $\mathrm{BaCO}_{3}$ (entspr. 0,031295 Grm. Ba).

$$
\begin{aligned}
& \text { Der Körper } \mathrm{C}_{16} \mathrm{H}_{19} \mathrm{~N}_{2} \mathrm{O}_{7} \mathrm{Ba}+2 \mathrm{H}_{2} \mathrm{O} \quad \text { Gefunden. } \\
& \text { enthält: } \quad 1 .
\end{aligned}
$$

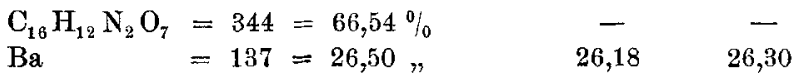

$$
\begin{aligned}
& 2 \mathrm{H}_{2} \mathrm{O} \quad=36=6,96 ", \quad-\quad 7,14 \text {. } \\
& 517 \quad 100,00
\end{aligned}
$$

Wie die angeführten Analysen zeigen, enthält der azoxyorthophenoxylessigsaure Baryt $2 \mathrm{Mol}$. Krystallwasser, von 
denen es durch Trocknen bei $120^{\circ}$ befreit werden kann. Es ist in heissem Wasser sehr schwer löslich und krystallisirt aus der erkaltenden Lösung in kurzen, das Licht stark doppelt brechenden Prismen. Essigsäure und stärkere Mineralsäuren lösen das Salz leicht auf.

Azoxyorthophenoxylessigsaures Aethyl,

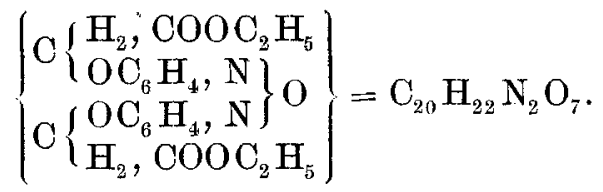

Schon oben bei der Beschreibung der Darstellungsmethode der Azoxyorthophenoxylessigsäure wurde erwähnt, dass der Aethyläther derselben leicht erhalten werden kann, wenn man in eine alkoholische Lösung genannter Säure trocknes Salzsäuregas einleitet. Dieselbe Verbindung kann auch dadurch gewonnen werden, dass man azoxyorthophenoxylessigsaures Silber in Alkohol suspendirt, eine genügende Menge Jodäthyl zusetzt und das Gemisch auf dem Wasserbade digerirt. Hierbei löst sich das verwendete Silbersalz nach und nach auf, und Jodsilber wird in gleichem Maasse abgeschieden. Sobald die Operation beendet ist, filtrirt man von letzterem ab und engt das Filtrat ein. Beim Erkalten der Flüssigkeit krystallisirt azoxyorthophenoxylessigsaures Aethyl aus.

Bei beiden Darstellungsmethoden ist es nicht unbedingt erforderlich, chemisch reine Azoxyorthophenoxylessigsäure anzuwenden. Ein Gemisch von solcher und Azoorthophenoxylessigsäure, wie es durch Reduction der O-Nitrophenoxylessigsäure mit Natriumamalgam leicht gewonnen werden kann, ist wegen der verschiedenen Löslichkeit der Aether beider Säuren immer dann gut zu gebrauchen, wenn es sich lediglich um Gewinnung von azoxyorthophenoxylessigsaurem Aethyl handelt.

\section{Analysen:}

1) $0,4990 \mathrm{Grm}$. bei $100^{\circ}$ getrockneter Substanz gaben, mit Kupferaxyd verbrannt, $30,0 \mathrm{Ccm}$. $\mathrm{N}$ bei $12^{\circ}$ und $768 \mathrm{Mm}$. Bar. (entsprechend 0,035994 Grm. N). 
2) 0,4702 Grm. bei $100^{\circ}$ getrockneter Substanz gaben, mit Kupferoxyd verbrannt, $28,7 \mathrm{Ccm} . \mathrm{N}$ bei $12^{\circ}$ und $754 \mathrm{Mm}$. Bar. (entspreehend 0,033797 Grm. N).

$$
\begin{array}{cccc}
\text { Die Substanz } \mathrm{C}_{20} \mathrm{H}_{22} \mathrm{~N}_{2} \mathrm{O}_{7} & \multicolumn{2}{c}{\text { Gefunden: }} \\
\text { enthält: } & 1 \text {. } & 2 . \\
\mathrm{N}=6,97 \% & 7,21 & 7,19
\end{array}
$$

Der Körper kann durch mehrmaliges Umkrystallisiren aus Alkohol und Behandeln mit Thierkohle chemisch rein erhalten werden. Er bildet alsdann lange, weisse Nadeln, welche Seideglanz besitzen. Sein Schmelzpunkt liegt bei $113^{0}-114^{0}$. Durch Behandeln mit Kalilauge gelingt es leicht, das azoxyorthophenoxylessigsaure. Aethyl in das Kalisalz überzuführen.

2. Azoorthophenoxylessigsäure,

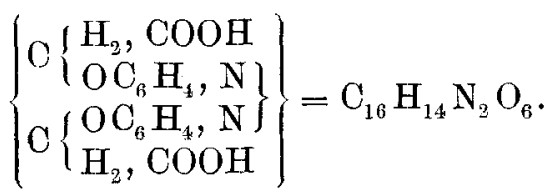

Azoorthophenoxylessigsäure bildet sich, wenn den beiden Nitrylen, welche in zwei Molekülen O-Nitrophenoxylessigsäure enthalten sind, dur'ch 8 At. nascirenden Wasserstoffs sämmtlicher Sauerstoff unter gleichzeitiger Bildung von $4 \mathrm{Mol}$. Wasser entzogen wird:

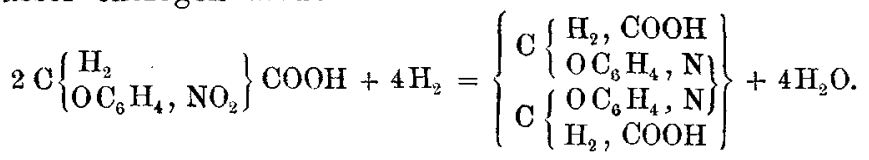

Genannte Säure entsteht ferner aus Azoxyorthophenoxylessigsäure dadurch, dass zwei Atome Wasserstoff sich mit dem den beiden Stickstoffatomen gemeinschaftlichen Atom Sauerstoff zu einem Molekül Wasser verbinden, ein Vorgang, welchen Gleichung II auf S. 152 ausdrückt.

Endlich liefert Oxydation von Hydrazoorthophenoxylessigsäure ebenfalls Azoorthophenoxylessigsäure.

Bei der Darstellung derselben wird in ganz analoger Weise wie bei derjenigen von Azoxyorthophenoxylessigsäure verfahren. In eine 15 proc. Lösung von 0 -nitrophenoxylJournal f. prakt. Chemie [2] Bd. 29. 
essiggaurem Natron wird ungefähr das $1-1^{1} / 3$ fache der theoretisch nöthigen Menge von Natriumamalgam nach und nach in kleinen Portionen eingetragen. Werden gleiche Quantitäten O-Nitrophenoxylessigsäure verarbeitet, wie sie bei der Darstellung der Azoxyorthophenoxylessigsäure angegeben wurden, so sind etwa 210-220 Theile 4proc. Amalgam erforderlich. Die Temperatur der Lösung kann während des Eintragens $60^{\circ}$ übersteigen, nur muss man auch hierbei durch Umschütteln für gehörige Mischung der sich roth färbenđen Flüssigkeit Sorge tragen. Nach Beendigung der Operation wird das ausgeschiedene Quecksilber entfernt, die beim Erkalten erstarrende Masse abgesaugt und in sehr wenig Wasser gelöst. Die Lösung ist zu filtriren und mit Essigsäure unter beständigem Umrühren anzusäuern. Salzsäure bewirkt zwar schnellere Ausscheidung der gebildeten Azoorthophenoxylessigsäure, jedoch ist diese weniger rein, als wenn sie mit Essigsäure gefällt wird.

In den meisten Fällen genügt es, die gewonnene Säure drei- bis viermal aus verdünntem Alkohol umzukrystallisiren, um sie in befriedigender Ausbeute chemisch rein zu erhalten. Einige Male dagegen war die Reduction nicht nach Wunsch verlaufen, ohne dass der Grund des Misserfolges sich mit wünschenswerther Sicherheit feststellen liess. Es wurde nämlich einerseits ein durch Azoxyorthophenoxylessigsäure stark verunreinigtes Produkt erhalten, bei dessen Bildung somit die Reduction auf einer zu frühen Stufe stehen geblieben war, andererseits schien in einem zweiten Falle die Reduction zu weit fortgeschritten zu sein, indem beim Ansäuern ausser der gewünschten Azoorthophenoxylessigsäure noch eine grau gefärbte Substanz ausfiel. Letztere ist, wie gleich im Voraus bemerkt werden soll, mit Hydrazoorthophenoxylessigsäure oder mit O-Amidophenoxylessigsäureanhydrid nicht identisch.

Sie unterscheidet sich von genannten Verbindungen vor allen Dingen durch ihre Unlöslichkeit, eine Eigenschaft, welche alle Versuche, sie zur Analyse chemisch rein zu erhalten, scheitern machte. Die Ursache der erwähnten Abweichungen dürfte ungleichmässig gemischtes Amalgam gewesen sein. 
Enthält die Azoorthophenoxylessigsäure bemerkenswerthe Mengen von Azoxyorthophenoxylessigsäure, so schmilat sie höher als $162^{\circ}$. Es würde alsdann unzweckmässig sein, sie durch Umkrystallisiren reinigen zu wollen, die Löslichkeitsverhältnisse beider Säuren sind einander sehr ähnlich. Die bei der Azoxyorthophenoxylessigsäure beschriebene Trennưng durch Ueberführung in die Aethyläther ist nicht anwendbar, wenn es sich um Reindarstellung der Azoorthophenoxylessigsäure handelt, weil ihr Aethyläther sehr schwer chemisch rein erhalten wird. Dagegen führt folgende Methode zum gewünschten Ziele: Man löst das Säuregemisch in heissem Wasser oder heissem, stark verdünnten Alkohol, setzt salpetersaures Silber zu und filtrirt den sich sofort ausscheidenden Niederschlag von azoorthophenoxylessigsaurem Silber heiss ab. Aus dem erkaltenden Filtrat scheidet sich ein Gemisch von wenig azoorthophenoxylessigsaurem Silber und den Silbersalzen der Azoxyorthophenoxylessigsäure aus. Der zuerst abfiltrirte Niederschlag wird mit heissem Wasser mehrere Male ausgezogen, in verdünntem warmen Alkohol suspendirt und durch Salzsäure zersetzt. Aus der vom Chlorsilber abfiltrirten Lösung krystallisirt beim Erkalten chemisch reine Azoorthophenoxylessigsäure aus.

Analysen:

1) 0,2775 Grm. Substanz, welche bis zu erlangter Gewichtseonstanz getrocknet worden waren, lieferten bein Verbrennen mit Kupferoxyd 0,5887 Grm. $\mathrm{CO}_{2}$ (entsprech. 0,1606 Grm. C) und 0,1076 Grm. $\mathrm{H}_{2} \mathrm{O}$ (entsprech. 0,0120 Grm. $\mathrm{H}$ ).

2) 0,2744 Grm. getrockneter Substanz gaben, mit Kupferoxyd verbrannt, 19,3 Ccm. N bei $8^{\circ}$ und $765 \mathrm{Mm}$. Bar. (entsprech. 0,0234688 Grm. N).

3) 0,1485 Grm. getrockneter Substanz lieferten beim Verbrennen mit Kupferoxyd 0,3170 Grm. $\mathrm{CO}_{y}$ (entsprech. 0,0864 Grm. C) und 0,0635 Grm. $\mathrm{H}_{2} \mathrm{O}$ (entsprech. 0,0070 Grm. H).

4) 0,1973 Grm. getrockneter Substanz lieferten, mit Kupferoxyd verbrannt, $15,0 \mathrm{Ccm}$. $\mathrm{N}$ bei $21^{\circ}$ und $752 \mathrm{Mm}$. Bar. (entspr. 0,016893 Grm. N).

5) 0,1698 Grm. aus Wasser umkrystallisirter lufttrockner Substanz verloren beim Trocknen bei $110^{\circ}$ bis zum constanten Gewicht 0,0165 Grm. $\mathrm{H}_{2} \mathrm{O}$.

6) 0,2399 Grm. aus Wasser umkryst. lufttrockner Substanz ver- 
164 Thate: Ueber die Einwirk. von Reductionsmitteln

loren beim Trocknen bei $110^{\circ}$ bis zum constanten Gewicht 0,0238 Grm. $\mathrm{H}_{2} \mathrm{O}$.

7) 0,5582 Grm. ans Alkohol umkryst. Substanz, welehe acht Tage über Chlorcalcium gestanden hatten, verloren beim Trocknen bei $110^{\circ}$ 0,0548 Grm. $\mathrm{H}_{2} \mathrm{O}$.

Die Substanz $\mathrm{C}_{16} \mathrm{H}_{14} \mathrm{~N}_{2} \mathrm{O}_{6}$ enthält:

$$
\begin{aligned}
& \mathrm{C}_{16}=192=58,18 \% \\
& \mathrm{H}_{14}=14=4,24 \% \\
& \mathrm{~N}_{2}=28=8,49 \% \\
& \mathrm{O}_{6}=\frac{96=29,09 \%}{330} 100,00
\end{aligned}
$$

Die Substanz $\mathrm{C}_{16} \mathrm{H}_{14} \mathrm{~N}_{2} \mathrm{O}_{6}+2 \mathrm{H}_{2} \mathrm{O}$

$$
\text { enthält: }
$$

$$
\begin{aligned}
& \dot{\mathrm{C}}_{16} \mathrm{H}_{14} \mathrm{~N}_{22} \mathrm{O}_{6}=330=90,16 \% \\
& 2 \mathrm{H}_{.2} \mathrm{O}=\frac{36=9,84,}{366}
\end{aligned}
$$

\begin{tabular}{cccc}
\multicolumn{4}{c}{ Gefunden: } \\
\hline $\begin{array}{c}\text { 1. } \\
57,87\end{array}$ & - & 3. & 4. \\
4,32 & - & 58,18 & - \\
- & 8,55 & - & 8,56 \\
- & - & - & -
\end{tabular}

Azoorthophenoxylessigsäure krystallisirt aus ihrer wässerigen oder verdünnten alkoholischen Lösung mit $2 \mathrm{Mol}$. Krystallwasser in prachtvollen orangefarbenen Nadeln, welche intensiven Seideglanz besitzen. Betrachtet man dieselben im polarisirten Licht, so zeigen sie bei gekreuzten Nicols starke Doppelbrechung und erscheinen goldgelb gefärbt. Die wasserfreie Säure sieht ziegelroth aus, beim Stehen an der Luft zieht sie Wasser an und verwandelt sich nach und nach wieder in die wasserhaltige, was äusserlich schon daran erkannt werden kann, dass die ziegelrothe in die goldgelbe Färbung übergeht. Chlorcalcium vermag der Azoorthophenoxylessigsäure das Krystallwasser nicht zu entziehen, Schwefelsäure thut dies nur zum Theil, Trocknen bei $110^{\circ}$ vollständig.

Die Säure schmilzt im Röhrchen bei $162^{\circ}$ und erstarrt wieder bei $157^{\circ}-156^{\circ}$. Erhitzt man sie vorsichtig auf Platinblech über der Flamme, so schmilzt sie zunächst zu einer schön weinrothen Flüssigkeit, zersetzt sich bei weiterem Erhitzen unter Ausstossen gelber Dämpfe und verbrennt schliesslich mit russender Flamme.

Ausser in Wasser und Alkohol ist die Substanz löslich 
in Aether, Alkalien und starken Säuren. Ihre Lösungen sind entweder gelb oder roth gefärbt. Diejenige in warmem Wasser vermag blaues Lackmuspapier zu röthen. Sie giebt mit salpetersaurem Silber einen zinnoberrothen, gelatinösen Niederschlag, welcher sich beim Erhitzen zusammenballt und die überstehende Flüssigkeit vollständig klar erscheinen lässt. Im Ueberschuss des Fällungsmittels ist derselbe in der Wärme etwas löslich. Essigsaures Blei bewirkt einen gelben, flockigen Niederschlag, welcher sich im Ueberschuss des Fällungsmittels schon in der Kälte löst.

Die Salze der Azoorthophenoxylessigsäure sind unschwer darzustellen; sie zeichnen sich durch lebhafte gelbe oder rothe Färbung und, mit Ausnahme des Silbersalzes, durch gutes Krystallisationsvermögen aus.

$$
\begin{aligned}
& \text { Azoorthophenoxylessigsaures Kali, }
\end{aligned}
$$

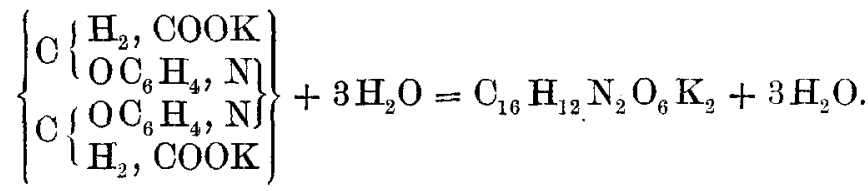

Zur Darstellung des azoorthophenoxylessigsauren Kalis löst man Azoorthophenoxylessigsäure in verdünnter reiner Kalilauge, indem man dafür sorgt, dass ein wenig Kali im Ueberschuss vorhanden bleibt. Die Lösung wird hierauf etwas eingeengt und mit so viel $99^{1} / 2$ proc. Alkohol versetzt, dass eben beginnende Trübung Krystallausscheidung ankündigt. Beim Erkalten krystallisirt neutrales azoorthophenoxylessigsaures $\mathrm{Kali}$ in orangefarbenen Blättchen aus, welche dem monoklinen oder triklinen System anzugehören scheinen. Um es zu reinigen, wird es abfiltrirt, in wenig Wasser gelöst und unter Zusatz von Alkohol auskrystallisiren gelassen.

\section{Analysen:}

1) $0,2425 \mathrm{Grm}$. lufttrockner Substanz verloren beim Trocknen bis zu erreichter Gewichtsconstanz bei $155^{\circ} 0,0285$ Grm. $\mathrm{H}_{2} \mathrm{O}$.

2) 0,1387 Grm. lufttrockner Substanz lieferten beim Abrauchen mit Sehwefelsäure 0,0517 Grm. $\mathrm{K}_{\mathbf{2}} \mathrm{SO}_{4}$ (entsprecb. 0,0232 Grm. K). 
166 Thate: Ueber die Einwirk. von Reductionsmitteln

3) 0,1657 Grm, lufttroekner Substanz verloren beim Trocknen bis zu erreichter Gewichtsconstanz bei $155^{\circ} 0,0195$ Grm. $\mathrm{H}_{2} \mathrm{O}$.

4) $0,1303 \mathrm{Grm}$. Iufttrockn. Substanz lieferten $0,0490 \mathrm{Grm} . \mathrm{K}_{2} \mathrm{SO}_{4}$ (entsprech. 0,0220 Grm. K).

5) 0,2140 Grm. wasserfr. Substanz (von Analyse 1) nahmen beim Stehen an der Tuft 0,0285 Grm. $\mathrm{H}_{2} \mathrm{O}$ auf.

6) 0,1462 Grm. wasserfreie Subst. (von Analyse 3) nahmen beim Stehen an der Luft 0,0195 Grm. $\mathrm{H}_{2} \mathrm{O}$ auf.

Der Körp. $\mathrm{C}_{16} \mathrm{H}_{12} \mathrm{~N}_{2} \mathrm{O}_{6} \mathrm{~K}_{2}+3 \mathrm{H}_{2} \mathrm{O}$ enthält:

Gefunden:

$$
\begin{aligned}
& \mathrm{C}_{16} \mathrm{H}_{12} \mathrm{~N}_{2} \mathrm{O}_{6}=328=71,30 \% \\
& \mathrm{~K}_{2} \quad=78=16,96 \text {, } \\
& 3 \mathrm{H}_{2} \mathrm{O}=\frac{54=11,74,}{460100,00} \\
& \begin{array}{rrrrrr}
1 . & 2 . & 3 . & 4 . & 5 . & 6 . \\
- & - & - & - & - & - \\
- & 16,73 & - & 16,88 & - & - \\
11,75 & - & 11,77 & - & 11,75 & 11,77
\end{array}
\end{aligned}
$$

Die Analysen zeigen, dass das azoorthophenoxylessigsaure Kali mit $3 \mathrm{Mol}$. Wasser krystallisirt. Letzteres wird durch Erhitzen auf $100^{\circ}$ nur theilweise entfernt; erst durch anhaltendes Trocknen bei $150^{\circ}$ gelingt es, den Körper wasserfrei zu erhalten. Er sieht alsdann roth aus und ist sehr hygroskopisch. Schon beim Stehen an der Luft werden von $\mathrm{ihm}$ genau $3 \mathrm{Mol}$. Wasser wieder aufgenommen. Die Substanz ist in Wasser in hohem Grade, in stark verdünntem Alkohol etwas weniger löslich. Die Löslichkeit wird durch vorhandenes freies Alkali bedeutend verringert. Starke Säuren setzen aus den Lösungen Azoorthophenoxylessigsäure in Freiheit. Die wässrige Lösung des Salzes reagirt neutral und giebt folgende Reactionen:

$\mathrm{BaCl}_{2}$ : rother, krystallinischer Niederschlag, welcher sich zu Boden setzt, in der Kälte erst nach einiger Zeit, beim Erwärmen sofort auftritt.

$\mathrm{Pb}\left(\mathrm{C}_{2} \mathrm{H}_{3} \mathrm{O}_{2}\right)_{2}$ : orangefarbener, flockiger Niederschlag, im Ueberschuss des Fällungsmittels beim Erwärmen und in Mineralsäuren löslich.

$\mathrm{AgNO} \mathrm{N}_{3}$ : zinnoberrother, gelatinöser Niederschlag, in Ammoniak und in Salpetersäure löslich, bei Zusatz von letzterer scheidet sich nach kurzer Zeit die freie Säure ab. $\mathrm{FeCl}_{3}$ : orangefarbener Niederschlag. $\mathrm{CuSO}_{4}$ : bräunlich gelber Niederschlag, im Ueberschuss des Fällungsmittels löslich. 
$\mathrm{HgCl}_{2}$ : rother Niederschlag, tritt erst nach starkem $\mathbf{K}_{0}$ chen auf.

$\mathrm{MgSO}_{4}$ : orangefarbener krystallinischer Niederschlag, in der Kälte erst nach einiger Zeit.

$$
\begin{gathered}
\text { Azoorthophenoxylessigsaures Natron, } \\
\left\{\begin{array}{l}
\mathrm{C}\left\{\begin{array}{l}
\mathrm{H}_{2}, \text { COONa } \\
\mathrm{OC}_{6} \mathrm{H}_{4}, \mathrm{~N} \\
\mathrm{OC}_{6} \mathrm{H}_{4}, \mathrm{~N}
\end{array}\right\} \\
\mathrm{H}_{2}, \mathrm{COONa}
\end{array}\right\}+3 \mathrm{H}_{2} \mathrm{O}=\mathrm{C}_{16} \mathrm{H}_{12} \mathrm{~N}_{2} \mathrm{O}_{6} \mathrm{Na}_{3}+3 \mathrm{H}_{2} \mathrm{O} .
\end{gathered}
$$

Die Darstellung und Reinigung dieses Salzes wird in ganz gleicher Weise vorgenommen, wie sie beim azoorthophenoxylessigsauren Kali angegeben wurde. In seinen Krystallformen und sonstigen Eigenschaften gleicht es dem Kalisalz vollkommen, nur ist es im wasserhaltigen Zustande mehr gelbroth als orange gefärbt.

Analysen:

1) 0,1142 Grm. lufttrockner Substanz lieferten nach Abrauchen mit Schwefelsäure 0,0382 Grm. $\mathrm{Na}_{2} \mathrm{SO}_{4}$ (entspr. 0,01237 Grm. Na).

2) 0,1248 Grm, lufttrockner Suhstanz verloren beim Trocknen bei $150^{\circ}-160^{\circ}$ bis zu erlangter Gewichtsconstanz $0,0160 \mathrm{Grm} . \mathrm{H}_{2} \mathrm{O}$.

$$
\begin{aligned}
& \text { Der Körper } \mathrm{C}_{16} \mathrm{H}_{12} \mathrm{~N}_{2} \mathrm{O}_{8} \mathrm{Na}_{2}+3 \mathrm{H}_{49} \mathrm{O} \text { Gefunden: } \\
& \text { enthält: } \quad 1.2 \text {. } 2 .
\end{aligned}
$$

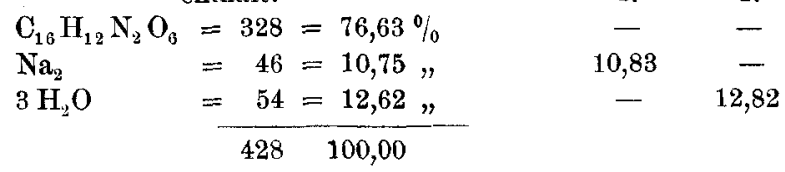

Azoorthophenoxylessigsaures Silber,

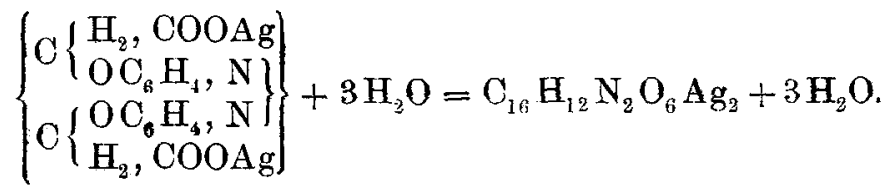

Schon oben bei der Beschreibung der Darstellangsmethode von Azoorthophenoxylessigsäure wurde erwähnt, dass deren Silbersalz direct durch Versetzen einer Lösung der Säure mit salpetersaurem Silber gewonnen werden kann. 
168 Thate: Ueber die Einwirk. von Reductionsmitteln

Handelt es sich um eine Reindarstellung desselben, so empfiehlt sich das angegebene Verfahren deswegen nicht, weil sich bei ihm neben dem neutralen Salz saures Salz zu bilden scheint. Es gelang zwar nicht, letzteres zu isoliren, doch lassen die angestellten Analysen diesen Schluss gerechtfertigt erscheinen. Zweckmässiger ist es, folgendermaassen zu verfahren: Eine verdünnte Lösung von azoorthophenoxylessigsaurem Kali oder Natron wird erhitzt und mit salpetersaurem Silber versetzt. Der entstehende zinnoberrothe Niederschlag, welcher sich zu Boden setzt, ist durch Decantiren mehrfach mit heissem Wasser auszuziehen, sodann auf einem Filter zu sammeln, abzusaugen und nach einander mit heissem Wasser, Alkohol und Aether zu waschen. Die letztgenannten Waschflüssigkeiten werden angewendet, um die sich fest zusammensetzende Substanz leichter lufttrocken zu erhalten.

Analysen:

1) 0,1422 Grm. lufttrockner Substanz verloren beim Trocknen bei $150^{\circ}-160^{\circ}$ bis zu erlangter Gewichtsconstanz $0,0137 \mathrm{Grm} . \mathrm{H}_{2} \mathrm{O}$ und lieferten beim Einäschern 0,0513 Grm. Ag.

2) 0,1190 Grm. Substanz, ebenso behandelt, verloren 0,0115 Grm. $\mathrm{H}_{2} \mathrm{O}$ und lieferten 0,0429 $\mathrm{Grm}$. Ag.

\begin{tabular}{|c|c|c|c|}
\hline Die Subst. $\mathrm{C}_{18} \mathrm{~F}$ & $\mathrm{H}_{12} \mathrm{~N}_{2} \mathrm{O}_{6} \mathrm{Ag}_{2}+3 \mathrm{H}_{2} \mathrm{O}$ & Gef & den: \\
\hline & nthält: & 1. & 2. \\
\hline $\mathrm{C}_{1 \mathrm{~s}} \mathrm{H}_{12} \mathrm{~N}_{2} \mathrm{O}_{6}$ & $=328=54,85 \%$ & - & - \\
\hline $\mathrm{Ag}_{2}$ & $=216=36,12$ & 36,08 & 36.05 \\
\hline $3 \mathrm{H}_{2} \mathrm{O}$ & $=54=9,03 \%$ & 9,63 & 9,66 \\
\hline & 100,00 & & \\
\hline
\end{tabular}

Der Körper ist amorph und im lufttrocknen Zustande mit 3 Mol. Wasser verbunden, welche schwer zu entfernen sind. Beim Erhitzen auf $100^{\circ}-105^{\circ}$ entweicht nur $1 \mathrm{Mol}$., der Rest erst bei $150^{\circ}-160^{\circ}$ vollständig, doch tritt hierbei sehr leicht Zersetzung ein. Dieselbe findet explosionsartig statt, wenn das Salz schnell über $170^{\circ}$ erhitzt wird. Seine Einäscherung muss deshalb zur Vermeidung eines Verlustes in gleicher Weise ausgeführt werden, wie sie bei dem azooxyorthophenoxylessigsaurem Silber angegeben wurde. Versuche, die Substanz umzukrystallisiren, gaben keine befriedigenden Resultate. Sie wurde zwar in kleinen, aus ziegel- 
rothen Nädelchen bestehenden Flocken krystallisirt erhalten, doch lieferten die damit angestellten Analysen immer zu grossen Silbergehalt. In Ammoniak ist das Salz leicht löslich, in den übrigen gebräuchlichen Lösungsmitteln, soweit sie nicht eine Zersetzung der Substanz verursachen, ist sie schwer löslich oder ganz unlöslich.

$$
\begin{gathered}
\text { A zoorthophenoxylessigsaurer Baryt, } \\
\left\{\begin{array}{l}
\mathrm{C}\left\{\begin{array}{l}
\mathrm{H}_{2}, \mathrm{COO} \\
\mathrm{OC}_{6} \mathrm{H}_{4}, \mathrm{~N} \\
\mathrm{OC}_{6} \mathrm{H}_{4}, \mathrm{~N} \\
\mathrm{H}_{2}, \mathrm{COO}
\end{array}\right\} \mathrm{Ba}
\end{array}\right\}+2 \mathrm{H}_{22} \mathrm{O}=\mathrm{C}_{16} \mathrm{H}_{12} \mathrm{~N}_{2} \mathrm{O}_{6} \mathrm{Ba}+2 \mathrm{H}_{2} \mathrm{O} .
\end{gathered}
$$

Um azoorthophenoxylessigsauren Baryt zu erhalten, versetzt man eine concentrirte Lösung des Natron- oder Kalisalzes mit Chlorbaryurn, saugt den entstehenden Niederschlag ab und krystallisirt ihn aus viel heissem Wasser um.

$$
\text { Analyse: }
$$

0,1511 Grm. lufttrockner Substanz verloren beim Trocknen bei $165^{\circ}$ bis za erlangter Gewichtsconstanz $0,0108 \mathrm{Grm} . \mathrm{H}_{2} \mathrm{O}$ und lieferten beim Einäschern 0,0583 Grm. $\mathrm{BaCO}_{3}$ (entspr. 0,040544 Grm. Ba).

Die Subst. $\mathrm{C}_{16} \mathrm{H}_{12} \mathrm{~N}_{2} \mathrm{O}_{6} \mathrm{Ba}+2 \mathrm{H}_{2} \mathrm{O}$ enthält: Gefunden:

$$
\begin{array}{llr}
\mathrm{C}_{16} \mathrm{H}_{12} \mathrm{~N}_{2} \mathrm{O}_{6} & =328=65,47 \% & - \\
\mathrm{Ba} & =137=27,34 \% & 26,83 \\
2 \mathrm{H}_{3} \mathrm{O} & =36=7,19 \% & 7,15
\end{array}
$$

Der Körper bildet kurze Prismen von gelbrother Farbe. Er ist in kaltem Wasser und verdünntem Alkohol wenig, in heissem Wasser etwas mehr löslich und krystallisirt mit 2 Mol. Wasser.

$$
\begin{aligned}
& \text { Azoorthophenoxylessigsaurer Kalk, } \\
& \left\{\begin{array}{l}
\mathrm{C}\left\{\begin{array}{l}
\mathrm{H}_{2}, \mathrm{COO} \\
\mathrm{OC}_{6} \mathrm{H}_{4}, \mathrm{~N} \\
\mathrm{OC}_{6} \mathrm{H}_{4}, \mathrm{~N} \\
\mathrm{H}_{2}, \mathrm{COO}
\end{array}\right\} \mathrm{Ca}
\end{array}\right\}+8 \mathrm{H}_{2} \mathrm{O}=\mathrm{C}_{16} \mathrm{H}_{12} \mathrm{~N}_{2} \mathrm{O}_{6} \mathrm{Ca}+8 \mathrm{H}_{2} \mathrm{O} .
\end{aligned}
$$

Aus einer Mischung concentrirter Lösungen von azoorthophenoxylessigsaurem Kali und Chlorcalcium scheidet 
170 Thate: Ueber die Einwirk. von Reductionsmitteln

sich das Kalksalz als rother Niedersehlag ab, welcher aus heissem Wasser umkrystallisirt werden kann.

\section{Analyse:}

0,2805 Grm. lufttrockner Substanz verloren beim Trocknen bei $185^{\circ}$ bis zu erlangter Gewichtsconstanz 0,0793 Grm. $\mathrm{H}_{2} \mathrm{O}$ und lieferten nach dem Einäschern 0,0310 Grm. CaO (entspr. 0,0221 Grm. Ca).

Die Subst. $\mathrm{C}_{16} \mathrm{H}_{12} \mathrm{~N}_{2} \mathrm{O}_{8} \mathrm{Ca}+8 \mathrm{H}_{2} \mathrm{O}$ enthält: Gefunden :

$$
\begin{array}{llr}
\mathrm{C}_{16} \mathrm{H}_{12} \mathrm{~N}_{2} \mathrm{O}_{6} & =328=64,06 \% & - \\
\mathrm{Ca} & =40=7,81 \% & 7,88 \\
8 \mathrm{H}_{2} \mathrm{O} & =144=28,13 \% & 28,27 \\
& 512 \quad 100,00 &
\end{array}
$$

Der Körper krystallisirt mit $8 \mathrm{Mol}$. Krystallwasser in prachtrollen goldrothen Nadeln. Dieselben scheinen dem monoklinen System anzugehören; es können Prismen, Domen und Pinakoïde gut an ihnen unterschieden werden.

Ausser in Wasser ist azoorthophenoxylessigsaurer Kalk auch in verdünntem Alkohol löslich.

Azoorthophenoxylessigsaures Aethyl,

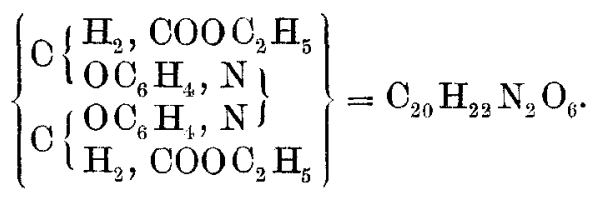

Wird in eine alkoholische Lösung von Azoorthophenoxylessigsäure trocknes Salzsäuregas eingeleitet, so bildet sich zwar azoorthophenoxylessigsaures Aethyl, doch scheint dasselbe von der überschüssigen Salzsäure wieder zersetzt zu werden. Aus der mit Salzsäure gesättigten Lösung vermochte Wasser nichts auszufällen, wenn dieselbe vorher nicht stark eingeengt wurde. Geschah dies, so setzte sich am Boden des Gefässes eine schmierige Masse $a b$, welche in Wasser unlöslich, in Alkohol leicht löslich war und nicht zur Krystallisation gebracht werden konnte. Die andere zur Aetherificirung angewendete Methode, in warmem Alkohol suspendirtes azoorthophenoxylessigsaures Silber durch Jodäthyl in Jodsilber und azoorthophenoxylessigsaures Aethyl uberzuführen, lieferte zwar keine guten, jedoch immerhin 
erfolgreiche Resultate. Die vom ausgeschiedenen Jodsilber abfiltrirte Lösung wurde eingeengt und die beim Erkalten ausgeschiedene Substanz mehrere Male aus Alkohol umkrystallisirt.

$$
\text { Analyse: }
$$

$0,1722 \mathrm{Grm}$. über Schwefelsäure getrockneter Substanz lieferten beim Verbrennen mit Kupferoxyd $11,3 \mathrm{Ccm}$. $\mathrm{N}$ bei $21^{\circ}$ und $749 \mathrm{Mm}$. Bar. (entsprech. 0,012674 Grm. N).

$$
\begin{array}{cc}
\text { Die Substanz } \mathrm{C}_{20} \mathrm{H}_{22} \mathrm{~N}_{2} \mathrm{O}_{6} \text { enthält: } & \text { Gefunden: } \\
\mathrm{N}=7,25 \% & 7,36
\end{array}
$$

Azoorthophenoxylessigsäure-Aethyläther krystallisirt in sehr unregelmässig gebildeten, rhomboëderähnlichen Formen, welche vielfach mit einander aggregirt sind und rothe Färbung besitzen. Er ist in kaltem Wasser unlöslich, in heissem Wasser ziemlich schwer und in Alkohol sehr leicht löslich. Durch verdünnte wässrige Kalilauge wird die Substanz, welche bei $110^{\circ}-111^{\circ}$ schmilzt, leicht in das Kalisalz übergeführt. Letzteres lieferte, mit Salzsäure zersetzt, freie Azoorthophenoxylessigsäure, deren Schmelzpunkt genau bei $162^{\circ}$ lag.

Auf Platinblech vorsichtig über der Flamme erhitzt, schmilat das azoorthophenoxylessigsaure Aethyl anfangs zu einer rothen Flüssigkeit, verflüchtigt sich jedoch sehr bald, ohne zu verbrennen, in gelben Dämpfen. Schnell erhitzt verbrennt es mit russender Flamme.

Die intensive rothe Färbung, mit welcher sich Azoortho- . phenoxylessigsäure in absolutem Alkohol löst, liess vermuthen, dass letztere beim Stehen der Lösung ätherificirt werde. Die hierüber angestellten Versuche gaben jedoch ein negatives Resultat. Die rothe Färbung der absolut-alkoholischen Säurelösung dürfte mithin wohl darin begrïndet sein, dass die Substanz in wasserfreiem Zustande sich gelöst befindet, in welchem sie, wie angegeben wurde, roth aussieht, und dass ihr Krystallwasser vom Alkohol gebunden wird. 
172 Thate: Ueber die Einwirk. von Reductionsmitteln

3. Hydrazoorthophenoxylessigsäure.

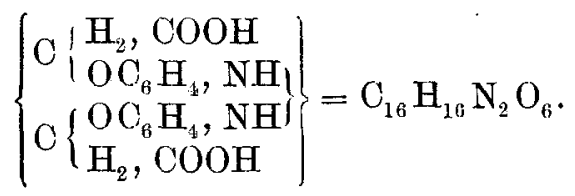

Die im Folgenden mitgetheilten Untersuchungen über Hydrazoorthophenoxylessigsäure sind keineswegs als abgeschlossen anzusehen, sie werden vielmehr in Verbindung mit denjenigen über das O-Amidophenoxylessigsäureanhydrid fortgesetzt.

Veranlassung hierzu bietet die weiter unten begründete Vermuthung, dass beide Substanzen in nahen Beziehungen zu einander stehen.

Um aus 2 Mol. O-Nitrophenoxylessigsäure $1 \mathrm{Mol}$. Hydrazoorthophenoxylessigsäure zu bilden, sind $10 \mathrm{At}$. Wasserstoff erforderlich. Acht derselben verbinden sich mit den 4 At. Sauerstoff der beiden Nitryle zu 4 Mol. Wasser, die beiden übrigen sättigen je eine Affinität des vorhandenen (als dreiwerthig angenommenen) Stickstoffs.

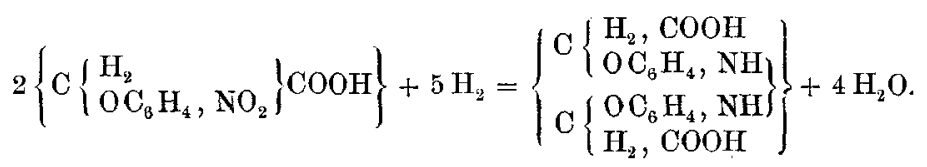

Aus einem Molekül Azoorthophenoxylessigsäure entsteht ein Molekül Hydrazoorthophenoxylessigsäure dadurch, dass sich 2 At. Wasserstoff mit dem vorhandenen Stickstoff direct verbinden, ein Vorgang, welchen Gleichung III auf Seite 152 ausdrückt.

Die Darstellung von Hydrazoorthophenoxylessigsäure ist mit Schwierigkeiten verknüpft, weil leichte Zersetzlichkeit, das Charakteristicum aller Hydrazoverbindungen, auch ihr eigen ist.

Durch Reduction der O-Nitrophenoxylessigsäure mit Natriumamalgam kommt man nur schwer zum Ziele. Es wurde deshalb nur durch einen Versuch die Thatsache con- 
statirt, dass sich die in Frage stehende Säure überhaupt dabei bildet. Bei den übrigen Versuchen dagegen diente Azoorthophenoxylessigsäure als Ausgangsmaterial.

Auch durch diese ist es noch nicht gelungen, grössere Quantitäten der freien Säure zu gewinnen, doch wurde deren Kalisalz und Barytsalz rein erhalten und der Analyse unterworfen.

Zu ihrer Darstellung kam folgendes Verfahren in Anwendung:

Eine Lösung von Azoorthophenoxylessigsäure in starkem alkoholischem Ammoniak wird mit Schwefelwasserstoff gesättigt. Die eintretende Reduction macht sich sehr bald dadurch sichtbar, dass die ursprünglich dunkel-weinrothe Färbung der Lösung heller wird. Man kann den Process durch Erwärmen beschleunigen, nur muss, wenn zum Sieden erhitzt wird, darauf Rücksicht genommen werden, dass immer Schwefelammonium im Ueberschuss bleibt. Im entgegengesetzten Falle tritt wiederum intensive Rothfärbung auf, ein Zeichen, dass die gebildete Hydrazoorthophenoxylessigsäure zu Azoorthophenoxylessigsäure oxydirt wird. Am vortheilhaftesten ist es, die kalt gesättigte Lösung in einem verschlossenen Kolben längere Zeit stehen zu lassen. Es scheidet sich alsdann ein Gemisch von hydrazoorthophenoxylessigsaurem Ammon und Schwefel aus. Dasselbe wird zur Entfernung des letzteren aus Alkohol umkrystallisirt. Versucht man hierauf durch verdünnte Essigsäure die freie Hydrazoorthophenoxylessigsäure auszufällen, so gelingt dies nur zum Theil. Die mit der gewonnenen Substanz angestellten Analysen zeigten, dass dieselbe im Wesentlichen saures hydrazoorthophenoxylessigsaures Ammon war. Sie wurde deshalb durch Behandeln mit verdünnter Kalilauge in das Kalisalz übergeführt, dieses aus starkem Alkohol umkrystallisirt und der Analyse unterworfen.

$$
\text { Analysen: }
$$

1) 0,1940 Grm. lufttrockner Substanz gaben beim Abrauchen mit Schwefelsäure 0,0710 Grm. $\mathrm{K}_{2} \mathrm{SO}_{4}$ (entspreeh. 0,03183 Grm. K).

2) $0,1850 \mathrm{Grm}$. lufttrockner Substanz verloren bei $145^{\circ} 0,0215$ Grm. $\mathrm{H}_{2} \mathrm{O}$. 
174 Thate: Ueber die Einwirk. von Reductionsmitteln

$$
\begin{array}{lrcc}
\text { Die Substanz } \mathrm{C}_{16} \mathrm{H}_{14} \mathrm{~N}_{2} \mathrm{O}_{6} \mathrm{~K}_{2}+3 \mathrm{H}_{2} \mathrm{O} & \multicolumn{2}{c}{\text { Gefunden: }} \\
\text { enthält: } & 1 . & 2 . \\
\mathrm{C}_{16} \mathrm{H}_{14} \mathrm{~N}_{2} \mathrm{O}_{6} & =330=71,43 \% & - & - \\
\mathrm{K}_{2} & =78=16,88, & 16,41 & - \\
3 \mathrm{H}_{2} \mathrm{O} & =54=11,69, & - & 11,62 \\
& =562 \quad 100,00 & &
\end{array}
$$

Hydrazoorthophenoxylsaures Kali bildet, aus Alkohol umkrystallisirt, hellgelbe Rhomboëder, welche deutliche rhomboëdrische Spaltbarkeit besitzen, stark doppelbrechend, in Wasser und Alkohol leicht löslich und in Aether unlöslich sind. Sie enthalten $3 \mathrm{Mol}$. Krystallwasser und geben dieses beim Trocknen bei $145^{\circ}$ ab. Gleichzeitig verwandelt sich jedoch die Substanz zum Theil in azoorthophenoxylessigsaures Kali und nimmt zinnoberrothe Färbung an.

Die wässrige Lösung des krystallisirten Salzes giebt mit Chlorbaryum, essigsaurem Blei und salpetersaurem Silber hell crêmefarbige Niederschläge, die des getrockneten Salzes dagegen Reactionen, welche deutlich erkennen lassen, dass man es mit einem Gemisch von azoorthophenoxylessigsaurem und hydrazoorthophenoxylessigsaurem. Kali zu thun hat. Versetzt man eine concentrirte wässrige Lösung des wasserhaltigen Salzes mit einigen Tropfen Eisessig, so scheidet sich eine weisse, in Wasser und Alkohol leicht lösliche Sub$\operatorname{stanz} a b$.

Dieselbe färbt sich beim Liegen an der Luft oberflächlich gelb, im Röhrchen erhitzt, zersetzt sie sich bei $225^{\circ}$ bis $227^{\circ}$, ohne zu schmelzen; auf Platinblech über der freien Flamme vorsichtig erhitzt, bläht sie sich, ebenfalls ohne zu schmelzen, unter Abscheidung einer schwer verbrennlichen Kohle stark auf. Da nur wenig Substanz zur Verfügung stand, konnte ihre Elementaranalyse bis jetzt nicht ausgeführt werden.

Anfänglich war versucht worden, aus einer alkoholischen Lösung von hydrazoorthophenoxylessigsaurem Ammon, wie sie durch Reduction der Azoorthophenoxylessigsäure mit alkoholischem Schwefelammonium gewonnen wird, die freie Säure durch Salzsäure auszufällen. Da keine Substanz ausfiel, wurde die mit Salzsäure versetzte Lösung auf dem 
Wasserbade eingeengt. Hierbei bildete sich azooxyorthophenoxylessigsaures Aethyl.

Versuche, die gewonnenen hydrazoorthophenoxylessigsauren Salze in wässriger Lösung durch Salzsäure zu zersetzen, lieferten ebenfalls Azoxyorthophenoxylessigsäure vom Schmelzpunkt $185^{\circ}-186^{\circ}$.

Es muss deshalb angenommen werden, dass Hydrazoorthophenoxylessigsäure durch freie Mineralsäuren in Azooxyorthophenoxylessigsäure übergeführt wird, während die durch den Sauerstoff der Luft verursachte Oxydation nur bis zur Bildung von Azoorthophenoxylessigsäure führt.

Durch Fällen der wässrigen Lösung des oben besprochenen hydrazoorthophenoxylessigsauren Kalis mit Chlorbaryum gelang es noch das Barytsalz zu gewinnen. Dasselbe bildet ein hell crêmefarbiges Pulver und ist mit $2 \mathrm{Mol}$. Wasser verbunden, welche beim Trocknen bei $130^{\circ}-140^{\circ}$ abgegeben werden. Die Substanz scheint sich jedoch hierbei zu zersetzen, da sie dunkle Färbung annimmt.

$$
\text { Analyse: }
$$

$0,2350 \mathrm{Grm}$. lufttrockner Substanz verloren beim Trocknen 0,0180 Grm. $\mathrm{H}_{2} \mathrm{O}$ und lieferten eingeäschert $0,0920 \mathrm{Grm} . \mathrm{BaCO}_{3}$ (entsprech. 0,0640 Grm, Ba).

Die Subst. $\mathrm{C}_{16} \mathrm{H}_{14} \mathrm{~N}_{2} \mathrm{O}_{6} \mathrm{Ba}+2 \mathrm{H}_{2} \mathrm{O}$ enthält: Gefunden:

$$
\begin{array}{llr}
\mathrm{C}_{18} \mathrm{H}_{14} \mathrm{~N}_{2} \mathrm{O}_{6} & =330=65,61 \% & - \\
\mathrm{Ba} & =137=27,23 \% & \mathbf{2 7 , 2 3} \\
2 \mathrm{H}_{2} \mathrm{O} & =36=7,16 \% & \mathbf{7 , 6 6}
\end{array}
$$

Für die weiteren Versuche, deren Ausführung gegenwärtig in Angriff genommen ist, handelt es sich zunächst darum, grössere Mengen von Hydrazoorthophenoxylessigsäure zu gewinnen. Die bisher erzielten Ausbeuten an solcher sind ausserordentlich schwankend und unbefriedigend.

4. O-Amidophenoxylessigsäure, $\mathrm{C}\left\{\begin{array}{l}\mathrm{H}_{2} \\ \mathrm{OC}_{6} \mathrm{H}_{4}, \mathrm{NH}_{2}\end{array}\right\} \mathrm{COOH}=\mathrm{C}_{8} \mathrm{H}_{9} \mathrm{NO}_{3}$.

Endprodukt der Reduction von O-Nitrophenoxylessigsäure mittelst Natriumamalgam ist $\mathrm{O}$-Amidophenoxylessig- 
176 Thate: Ueber die Einwirk. von Reductionsmitteln

säure. Ihre Bildung lässt sich durch die Annahme erklären, dass die beiden Atome Sauerstoff des Nitrils eines Moleküls O-Nitrophenoxylessigsäure gegen $2 \mathrm{At}$. Wasserstoff ausgetauscht werden, indem sie sich mit weiteren $4 \mathrm{At}$. Wasserstoff zu $2 \mathrm{Mol}$. Wasser verbinden. Hierbei geht der fünfwerthige Stickstoff in den dreiwerthigen über.

$$
\mathrm{C}\left\{\begin{array}{l}
\mathrm{H}_{2} \\
\mathrm{OC}_{6} \mathrm{H}_{4}, \mathrm{NO}_{2}
\end{array}\right\} \mathrm{COOH}+3 \mathrm{H}_{2}=\mathrm{C}\left\{\begin{array}{l}
\mathrm{H}_{2} \\
\mathrm{OC}_{6} \mathrm{H}_{4}, \mathrm{NH}_{2}
\end{array}\right\} \mathrm{COOH}+2 \mathrm{H}_{2} \mathrm{O} .
$$

$\mathrm{O}$-Amidophenoxylessigsäure scheint jedoch im freien $\mathrm{Zu}$ stande nicht bestehen zu können, wenigstens ist ihre Isolirung zur Zeit noch nicht gelungen. Sie spaltet sich vielmehr in $1 \mathrm{Mol}$. Wasser und einen Körper, welcher als O-Amidophenoxylessigsäureanhydrid bezeichnet werden kann, wenn im Namen sein Entstehungsprocess erklärend ansgedrückt werden soll:

$$
\underbrace{\mathrm{C}_{8} \mathrm{H}_{9} \mathrm{NO}_{3}}_{\begin{array}{c}
\text { O-Amidophenoxyl- } \\
\text { essigsäure }
\end{array}}-\mathrm{H}_{2} \mathrm{O}=\underbrace{\mathrm{C}_{8} \mathrm{H}_{7} \mathrm{NO}_{2}}_{\begin{array}{l}
\text { O-Amidophenoxyl- } \\
\text { essigsätureanhydrid }
\end{array}} .
$$

Die Substanz wird als Nebenprodukt bei der Darstellung der Azoorthophenoxylessigsäure in folgender Weise gewonnen:

Das Filtrat von dem nach Beendigung der Reduction ausgeschiedenen Natronsalze genannter Säure wird mit Salzsäure angesäuert und auf dem Wasserbade zur Trockne gebracht. Die so erhaltene Masse besteht aus viel Chlornatrium, Azoorthophenoxylessigsäure und O-Amidophenoxylessigsäureanhydrid. Zur Entfernung des Chlornatriums wird dieselbe pulverisirt und mit absolutem Alkohol ausgezogen. Die alkoholische Lösung ist abermals auf dem Wasserbade zur Trockne zu bringen, und der Rückstand mit viel lauwarmem Wasser zu extrahiren. Die wässrige Lösung enthält den grössten Theil des gebildeten O-Amidophenoxylessigsäureanhydrids neben wenig Azoorthophenoxylessigsäure. Zur Entfernung der letzteren wird sie stark eingeengt, die ausgeschiedene Krystallmasse in alkoholischer Lösung mit Thierkohle behandelt und schliesslich aus verdünntem Alkohol umkrystallisirt. Die so gewonnene Substanz besteht aus 
kurzen Prismen, welche im durchfallenden Lichte farblos, im reflectirten weiss erscheinen, bei $166^{\circ}-167^{\circ}$ schmelzen und sich in allen ihren Eigenschaften als identisch mit dem durch Reduction der O-Nitrophenoxylessigsäure mittelst Eisenfeile und Essigsäure zu erhaltenden Produkt erweisen.

Die ausführliche Beschreibung des Körpers erfolgt deshalb weiter unten. An dieser Stelle mögen nur die Ergebnisse der angestellten Analysen noch angeführt werden.

Analysen:

1) $0,2115 \mathrm{Grm}$. bei $100^{\circ}$ getrockneter Substauz lieferten, mit Kupferoxyd verbrannt, $0,5004 \mathrm{Grm}$. $\mathrm{CO}_{2}$ (entspr. 0,1365 Grm. C) und 0,0913 Grm. $\mathrm{H}_{2} \mathrm{O}$ (entspr. 0,0101 Grm. $\mathrm{H}$ ).

2) 0,1610 Grm. der nämlichen Substanz lieferten, mit Kupferoxyd verbrannt, $12,8 \mathrm{Ccm}$. N bei $8^{0}$ und $755 \mathrm{Mm}$. Bar. (entspreeh. 0,0154 Grm. N).

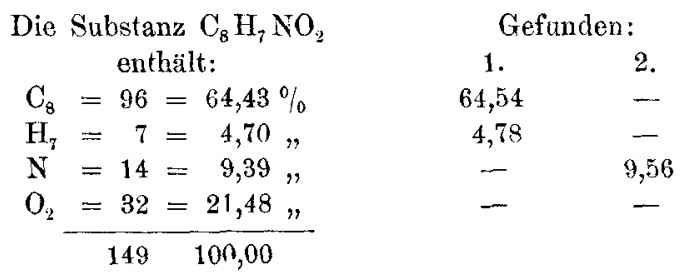

Zur Reduction der $\mathrm{O}$-Nitrophenoxylessigsäure in alkalisch reagirender Lösung kam ausser Natriumamalgam auch eine alkoholische Lösung von Aetznatron zur Verwendung. Die hiermit angestellten Versuche, durch welehe beabsichtigt wurde, Azoxyorthophenoxylessigsäure darzustellen, nahmen nicht den gewünschten Verlauf. Es zeigte sich, dass alkoholische Natronlauge sehr energisch einwirkt und weitgehende Umsetzungen veranlasst, deren Produkt eine ölige Flüssigkeit ist. Dieselbe ist mit Wasserdämpfen fliuchtig und sehr leicht zersetzlich, so dass alle Bemühungen, sie zur Analyse chemisch rein zu erhalten, fruchtlos waren. Thr Geruch gleicht vollkommen dem von Meum athamanticum und Levisticum officinale, doch konnte nicht bewiesen werden, ob sie mit dem Camphen genannter Pflanzen etwa identisch ist oder in Beziehungen irgend welcher Art zu demselben steht. 


\section{Thate: Ueber die Einwirk. von Reductionsmitteln}

B. Reduction der O-Nitrophenoxylessigsäure in sauer reagirenden Lösungen.

Für sauer reagirende Lösungen ist Natriumamalgam als reducirendes Mittel aus dem Grunde ungeeignet, weil bei seiner Verwendung immer ein grosser, nicht gut berechenbarer Ueberschuss von diesem Reagens erforderlich ist. Es wurden deshalb nur wenige Versuche damit unternommen. Der Erfolg derselben war insofern unbefriedigend, als es bei ihnen nicht, wie bei der Reduction in alkalischer Lösung, glückte, die von der $\mathrm{O}$-Nitrophenoxylessigsäure sich ableitenden Azoverbindungen in einfacher Weise zu isoliren.

Wird O-Nitrophenoxylessigsäure der Reduction durch Eisenfeile und Essigsäure unterworfen, so entsteht, wie schon mehrfach erwähnt wurde, O-A midophenoxylessigsäureanhydrid, wird dagegen eine salzsaure Lösung von Zinnchlorür als Reductionsmittel angewendet, so bildet sich stets entweder neben dem genannten Anhydrid oder ausschliesslich Monochlororthoamidophenoxylessigsäureanhydrid.

1. O-A midophenoxylessigsäureanhydrid, $\mathrm{C}_{8} \mathrm{H}_{7} \mathrm{NO}_{2}$.

Als beste Methode zur Darstellung von O-Amidophenoxylessigsäureanhydrid wurde die folgende erkannt:

O-Nitrophenoxylessigsäure wird mit Eisenfeilspähnen vermischt, das Gemisch mit 25\% Essigsäure in einem Kolben etwa eine Stunde lang auf dem Wasserbade erwärmt, lierauf mit Wasser verdünnt und noch warm von dem ungelösten Eisen, beziehentlich dem ausgeschiedenen Eisenhydroxyd abfiltrirt. Das Filtrat wird auf dem Wasserbade zur Trockne gebracht, der Rückstand mit Alkohol aufgenommen, die alkoholische Lösung verdunstet und der jetzt verbleibende Rückstand mit heissem Wasser ausgezogen. Beim Erkalten der eingeengten wässrigen Lösung scheidet sich O-Amidophenoxylessigsäureanhydrid krystallinisch ab. Sollte die so gewonnene Substanz nicht rein weiss, sondern noch durch Eisen verunreinigt sein, so wird sie nochmals in heissem Wasser gelöst und die filtrirte Lösung wiederum auf dem Wasserbade zur Trockne gebracht. Durch das 
wiederholte Eindampfen der Lösung wird bezweckt, die in ihr befindlichen Eisensalze unter Abscheidung von Eisenoxydhydrat zu zersetzen, ein Ziel, zu welchem man durch Kochen der Lösung allein nur schwierig gelangt. Schliesslich ist es vortheilhaft, den Körper durch Behandeln mit Thierkohle von den letzten Spuren verunreinigender Substanzen zu befreien.

Analyse:

$0,2530 \mathrm{Grm}$. bei $100^{\circ}$ getrockneter Substanz gaben, mit Kupferoxyd verbrannt, $0,6000 \mathrm{Grm}$. $\mathrm{CO}_{2}$ (entsprech. 0,16364 Grm. $\mathrm{O}=64,68$ pCt. C) und 0,1115 Grm. $\mathrm{H}_{2} \mathrm{O}$ (entspr. 0,0124 Grm. $\mathrm{H}=4,90 \% \mathrm{H}$ ). - Die Substanz $\mathrm{C}_{8} \mathrm{H}_{7} \mathrm{NO}_{3}$ enthält der Berechnung nach, wie schon auf Seite 177 angegeben wurde, $64,43 \% \mathrm{C}$ und $4,78 \% \mathrm{H}$ ).

O-Amidophenoxylessigsäureanhydrid krystallisirt aus verdünntem Alkohol in kurzen, würfelähnlichen Prismen, von parallelopipetischer Spaltbarkeit. Da dieselben im polarisirten Licht starke Doppelbrechung erkennen lassen, so ist die Vermuthung widerlegt, dass man es mit undeutlich ausgebildeten regulären Hexaëdern zu thun habe.

Aus viel Wasser umkrystallisirt, wird die Substanz in rein weissen, sichelförmig gekrümmten Nadeln erhalten, welche zuweilen bis ein Centimeter gross werden. Sie bestehen, wie ihre mikroskopische Betrachtung lehrt, aus vielen kurzen, treppenartig an einander gereihten Prismen.

Ausser in Wasser and Alkohol ist das O-Amidophenoxylessigsäureanhydrid in Aether, Benzol und Alkalien leicht löslich. Seine salpetersaure Lösung färbt sich beim Kochen gelb. Es schmilat bei $166^{\circ}-167^{\circ}$ und erstarrt wieder bei $144^{\circ}$, sublimirt jedoch schon bei $110^{\circ}$ in weissen, seideglänzenden Nadeln. Der Schmelzpunkt der sublimirten Substanz liegt ebenfalls bei $166^{\circ}-167^{\circ}$. Auf Platinblech erhitzt verbrennt die Substanz leicht vollständig. Es war nicht möglich, dieselbe durch anhaltendes Kochen mit Salzsänre vom. spec. Grew. 1,15 in Monochlororthoamidophenoxylessigsäureanhydrid überzuführen. Die hiervon abweichende Angabe in der vorläufigen Mittheilung über den behandelten Gegenstand $\left.{ }^{1}\right)$ hat darin ihren Grund, dass die zu den angeführten

1) Dies. Journ. [2] 25, 266. 
180 Thate: Ueber die Einwirk. von Reductionsmitteln

Versuchen verwendete Substanz nach den Angaben von Fritzsche dargestellt worden war und demaach schon das Anhydrid der gechlorten Säure enthielt.

Das O-Amidophenoxylessigsäureanhydrid zeigt in wässeriger Lösung weder saure noch basische Eigenschaften, vermag jedoch, mit Alkalien gekocht, Salze zu bilden. Dieselben sind im Allgemeinen ziemlich unbeständig und zerfallen, namentlich beim Erwärmen, leicht wieder in die Componenten, aus welchen sie gebildet wurden.

2. Salze der O-Amidophenoxylessigsäure.

$$
\begin{aligned}
& \text { O-Amidophenoxylessigsaures } \mathrm{Kali} \text {, } \\
& \mathrm{C}\left\{\begin{array}{l}
\mathrm{H}_{2} \\
\mathrm{OO}_{6} \mathrm{H}_{4}, \mathrm{NH}_{2}
\end{array}\right\} \text { COOK }=\mathrm{C}_{8} \mathrm{H}_{8} \mathrm{NO}_{3} \mathrm{~K} \text {. }
\end{aligned}
$$

Zur Darstellung dieses Salzes wird O-Amidophenoxylessigsäureanhydrid mit dem Zehnfachen der theoretisch nöthigen Menge Aetzkali (Lösung 1:11/2) in einem weiten Tiegel auf dem Wasserbade digerirt. Sobald die Masse krystallinisch zu erstarren beginnt, setzt man $991 / 2 \% \mathrm{Al}-$ kohol zu und lässt erkalten. Der entstehende Krystallbrei ist abzusangen und mit absolutem Alkohol zu waschen. Zur Reinigung wird das rohe Salz in möglichst wenig Wasser gelöst und der Lösung ätherhaltiger, $991 / 2$ proc. Alkohol bis zur eben beginnenden Trübung zugesetzt. Hierbei seheidet sich etwa vorhandenes kohlensaures Kali aus. Man filtrirt schnell $a b$ und fällt durch reinen Aether das gewünschte Kalisalz aus. Sollte die wässrige Lösung desselben noch nicht neutral, sondern alkalisch reagiren, so ist das nämliche Reinigungsverfahren nochmals zu wiederholen.

\section{Analyse:}

0,1140 Grm. bei $130^{\circ}$ getrockneter Substanz gaben beim Abrauchen mit Schwefelsäure 0,0485 Grm. $\mathrm{K}_{2} \mathrm{SO}_{4}$ (entspr. 0,0217 Grm. K).

Der Körper $\mathrm{C}_{8} \mathrm{H}_{3} \mathrm{NO}_{3} \mathrm{~K}$ enthält: Gefunden:

$$
\begin{aligned}
& \mathrm{C}\left\{\begin{array}{l}
\mathrm{H}_{2}, \mathrm{COO} \\
\mathrm{OC}_{6} \mathrm{H}_{4}, \mathrm{NH}_{2}
\end{array}=166=80,98 \%\right. \\
& =39=19,02 \%
\end{aligned}
$$


O-Amidophenoxylessigsaures Kali bildet weisse, anscheinend monokline oder trikline Prismen. Dieselben setzen sich beim Filtriren zu einem dichten, papierartigen Filz zusammen, welcher Seidenglanz besitzt. Sie sind in Wasser sehr leicht löslich, in absolutem Alkohol und Aether unlöslich.

Ihre wässrige Lösung zeigt folgende Reactionen:

$\mathrm{BaCl}_{2}$ : in der Kälte kein Niederschlag, beim Aufkochen setzt sich ein solcher, welcher weiss aussieht, auf dem Boden des Gefässes ab.

$\mathrm{Pb}\left(\mathrm{C}_{2} \mathrm{H}_{3} \mathrm{O}_{2}\right)_{2}$ : dicker, weisser, sich zu Boden setzender Niederschlag.

$\mathrm{AgNO}_{3}$ : dicker, weisser Niederschlag bei Verwendung concentrirter Lösung, aus verdünnten Lösungen erfolgt nach einiger Zeit krystallinische Ausscheidung.

$\mathrm{FeCl}_{3}$ : dunkelbrauner Niederschlag, welcher erst beim Erwärmen auftritt.

$\mathrm{CuSO}_{4}$ : krystallinischer grüner Niederschlag.

Essigsäure oder Salzsäure scheiden aus der Lösung des Kalisalzes O-Amidophenoxylessigsäureanhydrid aus.

$$
\begin{aligned}
& \text { O-Amidophenoxylessigsaures Blei, } \\
& \left\{\mathrm{C}\left\{\begin{array}{l}
\mathrm{H}_{2}, \mathrm{COO} \\
\mathrm{OO}_{6} \mathrm{H}_{i}, \mathrm{NH}_{2}
\end{array}\right\}_{2} \mathrm{~Pb}=\mathrm{C}_{16} \mathrm{H}_{16} \mathrm{~N}_{2} \mathrm{O}_{6} \mathrm{~Pb} .\right.
\end{aligned}
$$

Wird eine warme Lösung von 0 -amidophenoxylessigsaurem Kali mit einer ebenfalls erwärmten Lösung von essigsaurem Blei versetzt, so beginnt sofort Ausscheidung des Bleisalzes, bestehend aus weissen Nadeln, welche sich zu dichten Büscheln aggregiren. Man lässt erkalten, saugt ab, wäscht zunächst mit warmem und hierauf mit kaltem Wasser.

$$
\text { Analyse: }
$$

$0,1200 \mathrm{Grm}$. bei $110^{\circ}-115^{0}$ getrocknete Substanz gaben beim Abrauchen mit Schwefelsäure $0,0670 \mathrm{Grm}$. $\mathrm{PbSO}_{4}$ (entsprech. 0,0458 Grm. Pb).

$$
\begin{aligned}
& \text { Die Subst. } \mathrm{C}_{16} \mathrm{H}_{16} \mathrm{~N}_{2} \mathrm{O}_{6} \mathrm{~Pb} \text { enthält: Gefunden: } \\
& \left.\begin{array}{rl}
\left\{\mathrm { C } \left\{\mathrm{H}_{2}, \mathrm{COO}\right.\right. \\
\mathrm{OC}_{6} \mathrm{H}_{4}, \mathrm{NH}_{2}
\end{array}\right\}_{2}=332=61,60 \% \\
& =\frac{207}{539}=\frac{38,40}{100,00},
\end{aligned}
$$


182 Thate: Ueber die Einwirk. von Reductionsmitteln

O-Amidophenoxylessigsaures Blei ist in Essigsäure und Salpetersäure leicht löslich. Beim Erwärmen auf $120^{\circ}$ färbt es sich in Folge beginnender Zersetzung gelblichbraun, bei $130^{\circ}$ zersetzt es sich vollständig, indem O-Amidophenoxylessigsäureanhydrid aus dem Zersetzungsprodukt sublimirt.

$$
\begin{aligned}
& \text { O-Amidophenoxylessigsaures Silber, } \\
& \mathrm{C}\left\{\begin{array}{l}
\mathrm{H}_{2} \\
\left.\mathrm{OC}_{6} \mathrm{H}_{4}, \mathrm{NH}_{2}\right\} \text { COOAg }=\mathrm{C}_{8} \mathrm{H}_{8} \mathrm{NO}_{3} \mathrm{Ag} .
\end{array}\right.
\end{aligned}
$$

Das Silbersalz wird durch Fällen einer Lösung des Kalisalzes mit salpetersaurem Silber erhalten. Es ist in warmem Wasser und in verdünntem warmen Alkohol leicht löslich. Seine salpetersaure Lösung ist in der Kälte kirschroth, beim Erhitzen färbt sie sich heller, bis sie orangefarben ist. Die Lösung der Substanz in Schwefelsäure sieht dunkel violett aus; diejenige in Ammoniak färbt sich ziemlich schnell braun.

Die Substanz ist ansserordentlich leicht zersetzlich. Die Zersetzung beginnt schon beim Abfiltriren und schreitet, auch wenn Tageslicht ausgeschlossen ist, rasch vorwärts. Durch Einwirkung von Licht und Wärme wird sie stark beschleunigt.

Das Salz konnte seiner grossen Zersetzlichkeit wegen nicht analysirt werden.

3. Monochlororthoamidophenoxylessigsäureanhydrid, $\mathrm{C}_{8} \mathrm{H}_{6} \mathrm{ClNO}_{2}$.

Das Monochlor-Orthoamidophenoxylessigsäureanhydrid steht zur Monochlororthoamidophenoxylessigsäure in gleichem Verhältniss wie das O-Amidophenoxylessigsäureanhydrid zur O-Amidophenoxylessigsäure.

Seine Bildung aus O-Nitrophenoxylessigsäure bei der Reduction mittelst Zinnchlorür in salzsaurer Lösung scheint in der Weise zu erfolgen, dass zunächst O-Amidophenoxylessigsäureanhydrid entsteht und dass sich dieses erst in einem zweiten Stadium in das Anhydrid der einfach gechlorten Säure umsetzt. Die Darstellung dieser Verbindung ist leicht auszuführen: 
O-Nitrophenoxylessigsäure in warmer, wässriger Lösung wird mit einer Lösung von Zinnchlorür in mässig starker Salzsäure im Ueberschuss vermengt, und die Mischung auf dem Wasserbade digerirt. Nach kurzer Zeit beginnt die Ausscheidung eines weissen krystallinischen Körpers, so dass, falls die angewandten Lösungen nicht zu verdünnt waren, die ganze Masse breiig erstarrt. Man lässt erkalten, saugt ab, krystallisirt eine Probe aus Wasser oder verdünntem Alkohol um und prüft, ob der Schmelzpunkt der Substanz ungefähr bei $190^{\circ}$ liegt. Kleine Abweichungen hiervon schaden nichts, da immer erst nach mehrmaligem Umkrystallisiren chemisch reine, bei $196^{\circ}-197^{\circ}$ schmelzende Substanz erhalten wird. Ergiebt sich der gewünschte Schmelzpunkt, so krystallisirt man die ganze Substanz aus verdünntem Alkohol um; sollte jedoch ein zu niedriger Schmelzpunkt gefunden worden sein, so ist es von Vortheil, die Substanz noch eine Zeit lang mit der Reductionsflüssigkeit zu behandeln.

\section{Analysen:}

1) 0,2642 Grm. Substanz lieferten, mit Kupferoxyd und vorgelegtem chromsauren Blei verbrannt, 0,5097 Grm. $\mathrm{CO}_{2}$ (entspr. 0,1390 Grm. C) und 0,0838 Grm. $\mathrm{H}_{2} \mathrm{O}$ (entspr. 0,0093 Grm. H).

2) $0,2110 \mathrm{Grm}$. Substanz lieferten, mit Kupferoxyd verbrannt, 14,6 Ccm. $\mathrm{N}$ bei $24^{\circ}$ und $755 \mathrm{Mm}$. Bar. (entspr. 0,01626 Grm. N).

3) 0,1545 Grm. Substanz, mit Kalk geglïht, lieferten $0,1178 \mathrm{Grm}$. $\mathrm{AgCl}$ (entspr. 0,0292 Grm. Cl).

4) 0,1520 Grm. Substanz, mit einem Gemisch von Kupferoxyd und chromsaurem Blei verbrannt, lieferten 0,2920 Grm. $\mathrm{CO}_{2}$ (entspr. 0,0796 Grm. C) und 0,0515 Grm. $\mathrm{H}_{2} \mathrm{O}$ (entspr. 0,0057 Grm. H).

5) 0,2833 Grm. Substanz, mit Kalk geglüht, lieferten 0,2187 Grm. $\mathrm{AgCl}$ (entspr. 0,0541 Grm. Cl).

Die Subst. $\mathrm{C}_{8} \mathrm{H}_{8} \mathrm{ClNO}_{2}$ enthält:

$\mathrm{C}_{8}=96=52,32 \%$

$\mathrm{H}_{0}=6=3,27$,

$\mathrm{Cl}=35,5=19,35$,

Gefunden.

$\mathrm{N}=14=7,63$,

$\mathrm{O}_{2}=32=17,43$,

$183,5 \quad 100,00$

\begin{tabular}{ccccc}
\hline 1. & 2. & 3. & 4. & 5. \\
52,61 & - & - & 52,36 & - \\
3,52 & - & - & 3,75 & - \\
- & - & 18,90 & - & 19,10 \\
- & 7,71 & - & - & - \\
- & - & - & - & -
\end{tabular}

Monochlororthoamidophenoxylessigsäureanhydrid bildet. 


\section{$18+$ Thate: Ueber die Einwirk. von Reductionsmitteln}

feine, vielfach verästelte Nadeln, welche im reflectirten Licht rein weisse Färbung und Seidenglanz erkennen lassen. Dieselben sind in kaltem Wasser fast gar nicht, in heissem Wasser, Aether und Benzol schwer, in starkem Alkohol ziemlich leicht löslich. Sie schmelzen bei $196^{\circ}-197^{\circ}$ und sublimiren bei etwa $130^{\circ}$. Das in der Substanz enthaltene Chlor kann erst dann nachgewiesen werden, wenn dieselbe durch Glühen mit Kalk zerstört wurde. Durch Behandeln mit concentrirter Kalilauge (Lösung 1:1) gelingt es nicht, dasselbe aus der Verbindung zu eliminiren. Trägt man den Körper in kleinen Portionen in schmelzendes Aetzkali ein, so findet eine äusserst energische Reaction statt, welche mit einer tiefgreifenden Zersetzung verbunden ist, wie an der Abscheidung von Kohle erkannt werden kann.

Im Uebrigen besitzt die Substanz grosse Aehnlichkeit mit dem O-Amidophenoxylessigsäureanhydrid. Sie zeigt, wie dieses, in heisser wässriger Lösung weder saure noch basische Eigenschaften, trotzdem gelingt es leicht, aus ibr durch Behandeln mit starken Lösungen von Alkalien monochloramidophenoxylessigsaure Salze darzustellen.

Freie Monochlororthoamidophenoxylessigsäure konnte nicht isolirt werden, doch ist anzunehmen, dass sie in wässeriger oder verdünnter saurer Lösung zu bestehen vermag. Versetzt man nämlich eine kalte Lösung von monochlororthoamidophenoxylessigsaurem Kali mit Essigsäure oder Salzsäure, so tritt erst nach geraumer Zeit Abscheidung von Monochlororthoamidophenoxylessigsäureanhydrid ein, während man erwarten sollte, dass dieselbe wegen der ausserordentlich geringen Löslichkeit letztgenannter Verbindung sofort erfolgen müsste.

4. Salze der Monochlororthoamidophenoxylessigsäure.

Monochlororthoamidophenoxylessigsaures Kali.

$\mathrm{C}\left\{\begin{array}{l}\mathrm{H}_{2} \\ \mathrm{OC}_{8} \mathrm{H}_{3} \mathrm{Cl}, \mathrm{NH}_{2}\end{array}\right\} \mathrm{COOK}=\mathrm{C}_{8} \mathrm{H}_{7} \mathrm{ClNO}_{3} \mathrm{~K}$.

Diese Verbindung wird aus dem Monochlororthoamidophenoxylessigsäureanhydrid in derselben Weise dargestellt 
und rein erhalten, wie auf S. 180 bei Beschreibung der Darstellung des O-amidophenoxylessigsauren Kalis geschildert wurde. Der Körper bildet rein weisse Krystallnadeln, welche sich bündelförmig aggregiren und getrocknet Seideglanz besitzen. Er ist in Wasser leicht, in Alkohol schwer löstich, in Aether unlöslich. Durch Einleiten von Kohlensäure in die alkoholische Lösung gelingt es nicht, Abscheidung von kohlensaurem Kali herbeizuführen, eine Thatsache, welche beweist, dass Kohlensäure das Salz nicht zu zersetzen vermag.

Die wässrige Lösung desselben reagirt neutral und zeigt folgende Reactionen:

$\mathrm{BaCl}_{2}$ : in der Kälte kein Niederschlag, nach dem Aufkochen und längeren Stehen erfolgt schwache Trübung.

$\mathrm{Pb}\left(\mathrm{C}_{2} \mathrm{H}_{3} \mathrm{O}_{2}\right)_{2}$ : weisser, krystallinischer Niederschlag. Derselbe ist in Essigsäure schon in der Kälte, im Ueberschuss des Fällungsmittels beim Erwärmen löslich.

$\mathrm{AgNO}_{3}$ : weisser, flockiger Niederschlag, löst sich in Ammoniak und fällt, wenn die Lösung vorsichtig mit Salpetersäure neutralisirt wird, wieder aus.

$\mathrm{FeCl}_{3}$ : dunkel weinrothe Färbung, nach einiger Zeit tritt ein sehr fein vertheilter kirschrother Niederschlag auf. $\mathrm{CuSO}_{4}$ : gelb lich-grüner Niederschlag. Derselbe löst sich bei vorsichtigem Erwärmen auf und scheidet sich beim Erkalten in feinen Krystallnadeln wieder ab. Mit Wasser gekocht, zersetzt er sich unter Abscheidung rothbrauner Flocken.

\section{Analyse:}

$0,1448 \mathrm{Grm}$. bei $115^{\circ}$ getrockneter Substanz lieferten beim Abrauchen mit Schwefelsäure $0,0524 \mathrm{Grm} \mathrm{K}_{2} \mathrm{SO}_{4}$ (entsprechend 0,0235 Grm. K).

Die Substanz $\mathrm{C}_{8} \mathrm{H}_{7} \mathrm{ClNO}_{3} \mathrm{~K}$ enthält:

Gefunden:

$$
\begin{aligned}
\mathrm{C}\left\{\begin{array}{l}
\mathrm{H}_{2}, \mathrm{COO} \\
\mathrm{OC}_{6} \mathrm{H}_{3} \mathrm{Cl}, \mathrm{NH}_{2}
\end{array}\right. & =200,5=83,72 \% \\
& =\frac{39}{239,5}=16,28,100,00
\end{aligned}
$$


186 Thate: Ueber die Einwirk. von Reductionsmitteln

Monochlororthoamidophenoxylessigsaures Natron,

$$
\mathrm{O}\left\{\begin{array}{l}
\mathrm{H}_{2} \\
\mathrm{OC}_{6} \mathrm{H}_{3} \mathrm{Cl}, \mathrm{NH}_{2}
\end{array}\right\} \mathrm{COONa} \text {. }
$$

Das Natronsalz wurde in gleicher Weise wie das Kalisalz gewonnen. Es gleicht diesem vollkommen und ist nur etwas schwieriger chemisch rein zu erhalten, da es in Alkohol leichter löslich ist als die entsprechende Kaliverbindung.

Monochlororthoamidophenoxylessigsaures Silber,

$$
\mathrm{C}\left\{\begin{array}{l}
\mathrm{H}_{2} \\
\mathrm{OO}_{6} \mathrm{H}_{3} \mathrm{Cl}, \mathrm{NH}_{2}
\end{array}\right\} \mathrm{COOAg}=\mathrm{C}_{3} \mathrm{H}_{7} \mathrm{ClNO}_{3} \mathrm{Ag} \text {. }
$$

Wird monochlororthoamidophenoxylessigsaures Kali in Alkohol gelöst und mit einer wässrigen Lösung von salpetersaurem Silber versetzt, so scheidet sich das Silbersalz sofort als voluminöser, mikrokrystallinischer Niederschlag ab. Man lässt denselben absetzen, decantirt mit verdinnntem Alkohol, saugt schnell ab, wäscht mit Aether und trocknet über Schwefelsäure bei Ausschluss von Licht.

\section{Analyse:}

0,1327 Grm. Substanz lieferten beim Einäschern 0,0465 Grm. Ag.

Die Substanz $\mathrm{C}_{8} \mathrm{H}_{7} \mathrm{Cl} \mathrm{NO}_{3} \mathrm{Ag}$ enthält:

Gefunden:

$$
\begin{aligned}
& \mathrm{C}\left\{\begin{array}{l}
\mathrm{H}_{2}, \mathrm{COO} \\
\mathrm{OC}_{6} \mathrm{H}_{3} \mathrm{Cl}, \mathrm{NH}_{2}=200,5=64,99 \% \\
=108=35,01,
\end{array}\right. \\
& \mathrm{Ag} \quad \frac{308,5}{100,00}
\end{aligned}
$$

Die Substanz bildet ausserordentlich kleine, weisse Krystalle, welche sich bei Anwendung von polarisirtem Licht und starker Vergrösserung als doppelt brechend erkennen lassen. Sie ist am Licht sehr leicht zersetzlich. Begünstigt wird die Zersetzung, wenn das Salz feucht ist. Sie findet sofort statt beim Kochen mit Wasser, Uebergiessen mit Natronlauge und Erwärmen auf $110^{\circ}$. Wird monochlororthoamidophenoxylessigsaures Silber vorsichtig auf $130^{\circ}$ erhitzt, so sublimirt aus seinem Zersetzungsprodukt Monochlororthoamidophenoxylessigsäureanhydrid in schönen, seideglänzenden Nadeln. 
Monochlororthoamidophenoxylessigsaures Blei, $\left(\mathrm{C}\left\{\begin{array}{l}\mathrm{H}_{2} \\ \mathrm{OC}_{6} \mathrm{H}_{3} \mathrm{Cl}, \mathrm{NH}_{2}\end{array}\right\} \mathrm{COO}\right)_{2} \mathrm{~Pb}=\mathrm{C}_{16} \mathrm{H}_{14} \mathrm{Cl}_{2} \mathrm{~N}_{2} \mathrm{O}_{6} \mathrm{~Pb}$.

Zur Darstellung des Bleisalzes wurde eine wässrige Lösung des Kalisalzes mit essigsaurem Blei gefällt, der Niederschlag mit warmem Wasser und Alkohol ausgewaschen und über Schwefelsäure getrocknet.

Analyse:

0,1270 Grm. Substanz lieferten, mit Schwefelsäure abgeraucht, 0,0630 Grm. $\mathrm{Pb} \mathrm{SO}_{4}$ (entspr. 0,04303 Grm. Pb).

Die Substanz $\mathrm{C}_{16} \mathrm{H}_{14} \mathrm{Cl}_{2} \mathrm{~N}_{2} \mathrm{O}_{6} \mathrm{~Pb}$ enthält: Gefunden:

$$
\begin{aligned}
2\left(\mathrm{C}\left\{\begin{array}{l}
\mathrm{H}_{2} \\
\mathrm{OC}_{3} \mathrm{H}_{3} \mathrm{Cl}, \mathrm{NH}_{2}
\end{array}\right\} \mathrm{COO}\right) & =401=65,95 \% \\
& =207=34,05,
\end{aligned}
$$

Der Körper bildet ein weisses, krystallinisches Pulver, er ist in kaltem Wasser, Allsohol und Aether unlöslich, in heissem Wasser schwer löslich. Erhitzt man die Substanz auf $130^{\circ}$, so sublimirt aus dem sich bildenden Zersetzungsprodukt Monochlororthoamidophenoxylessigsäureanhydrid.

\section{Schlussbetrachtung.}

Der von Fritzsche durch Reduction der O Nitrophenoxylessigsäure dargestellte Körper ist weder mit dem O-Amidophenoxylessigsäureanhydrid, noch mit dem Monochlororthoamidophenoxylessigsäureanhydrid identisch. Da die genannten Verbindungen in ihrem chemischen Verhalten grosse Aehnlichkeit zeigen, so könnte man glauben, genannter Autor habe es mit einem Gemenge beider zu thun gehabt. Dieser Annahme widerspricht jedoch die Thatsache, dass der Schmelzpunkt der von Fritzsche dargestellten Substanz bei $143^{\circ}$ bis $144^{\circ}$ liegt, während das O-Amidophenoxylessigsäureanhydrid bei $166^{\circ}-167^{\circ}$, das Monochlororthoamidophenoxylessigsäureanhydrid bei $196^{\circ}-197^{\circ}$ und Gemenge beider zwischen $166^{\circ}-197^{\circ}$ schmelzen.

Sehr leicht gelang es, durch Versuche nachzuweisen, dass 
188 Thate: Ueber die Einwirk. von Reductionsmitteln

durch Reduction der O-Nitrophenoxylessigsäure mit Zinnchlorür und Salzsäure sich immer das Anhydrid der gechlorten Säure bildet und selbst dann schon vorhanden ist, wenn die Reduction vor ihrer Beendigung unterbrochen wird. Sehr leicht gelang es auch, Produkte zu erhalten, deren Analyse mit Sicherheit den Schluss rechtfertigt, dass man es bei unvollendeter Reduction häufig mit einem Gemenge von O-Nitrophenoxylessigsäure, O-A midophenoxylessigsäureanhydrid und Monochlororthoamidophenoxylessigsäureanhydrid zu thun hat, jedoch nur in zwei von 19 Versuchen, welche in dieser Richtung unternommen wurden, glückte es, Substanzen zu isoliren, welche den Schmelzpunkt $143^{\circ}-144^{\circ}$ zeigten

Obgleich nun bei sämmtlichen Versuchen immer mit genau abgewogenen Mengen, unter Beobachtung der Temperatur und Concentration der Lösung und genauer Bestimmung der Dauer des Versuchs gearbeitet wurde, so ist es doch nicht möglich gewesen, die Bedingungen festzustellen, unter denen sich die bewusste Verbindung mit dem Schmelzpunkt $143^{\circ}-144^{\circ}$ bildet. Es findet dies seine Erklärung darin, dass jene ausserordentlich leicht zersetzlich ist. Schon durch einmaliges Umkrystallisiren, namentlich wenn dabei die Lösung zum Sieden erhitzt wird, und noch mehr durch Sublimation ändert sich ihr Schmelzpunkt oft um zehn und noch mehr Grade, immer jedoch steigt derselbe, niemals wird er niedriger, und immer bekommt man dann bei seiner Bestimmung den Eindruck, als ob man es jetzt mit einem Gemenge zu thun habe, während vorher die Substanz einheitlich schmolz.

Von vornherein musste deswegen darauf verzichtet werden, den Körper zur Vornahme analytischer Bestimmungen durch Umkrystallisiren zu reinigen. Die lufttrockne Substanz, wie sie durch den Versuch gewonnen worden war, besass einen Gehalt an Stickstoff von $8,5 \%$ und einen solchen an Chlor von 8,9\%. Ein Körper, welcher aus einem Molekül O-Amidophenoxylessigsäureanhydrid und einem Molekül Monochlororthoamidophenoxylessigsäureanhydrid zu- 
sammengesetzt ist, müsste nach der Berechnung $8,4 \% \mathrm{~N}$ und $10,7 \% \mathrm{Cl}$ enthalten.

Da die gefundenen Procentzahlen von den berechneten nicht allzu sehr abweichen und, wie oben gezeigt wurde, bei der Reduction mit Zinnchlorür sich O-Amidophenoxylessigsäureanhydrid neben Monochlororthoamidophenoxylessigsäureanhydrid bildet, so ist der Schluss nicht ganz unbegründet, dass die bei $143^{\circ}-144^{\circ}$ schmelzende Substanz in der That aus einem Molekül O-Amidophenoxylessigsüureanhydrid and einem Molekül Monochlororthoamidophenoxylessigsäureanhydrid besteht, also die Zusammensetzung hat:

$$
\left(\mathrm{C}_{8} \mathrm{H}_{7} \mathrm{NO}_{2}+\mathrm{C}_{8} \mathrm{H}_{6} \mathrm{ClNO}_{2}\right) \text {. }
$$

Wenn es aber möglich ist, dass derartige gepaarte Verbinđungen überhaupt bestehen können, so dürfte es auch gerechtfertigt sein, anzunehmen, dass nicht nur je zwei Moleküle verschiedener "Anhydride", sondern auch je zwei Moleküle desselben "Anhydrids" sich zu vereinigen vermögen, dass, mit anderen Worten, die Moleculargewichte der beschriebenen "Anhydride" in Wirklichkeit doppelt so gross sind, als sie angenommen wurden. Nicht:

sondern :

$$
\mathrm{C}_{8} \mathrm{H}_{7} \mathrm{NO}_{2} \text { und } \mathrm{C}_{8} \mathrm{H}_{6} \mathrm{ClNO}_{2} \text {, }
$$

$$
\mathrm{C}_{16} \mathrm{H}_{14} \mathrm{~N}_{2} \mathrm{O}_{4} \text { und } \mathrm{C}_{16} \mathrm{H}_{12} \mathrm{Cl}_{2} \mathrm{~N}_{2} \mathrm{O}_{4}
$$

wären demnach deren richtige empirische Formeln. Durch den directen Versuch lässt sich diese Frage deswegen nicht entscheiden, weil die Zersetzlichkeit der zu untersuchenden Verbindungen ihre Dampfdichtebestimmung unmöglich macht. Sollte es jedoch auf anderem Wege gelingen, darüber Aufklärung zu erhalten, so dürfte, falls die geäusserte Ansicht sich als richtig erweist, ein weiterer Schritt zur Erklärung der Constitution der sogenannten „inneren Anhydride" gethan sein. Die Stellung dieser zwischen den Hydrazoverbindungen und Amidoverbindungen einerseits und die grosse Veränderlichkeit der ersteren andererseits gestatten es, die „inneren Anbydride“ von den Hydrazoverbindungen abzuleiten. So könnte man sich z. B. vorstellen, dass das O-Amidophenoxylessigsäureanhydrid aus der Hydrazoortho- 
190 Thate: Ueber die Einwirk. v. Reductionsmitteln etc.

phenoxylessigsäure durch Abgabe der beiden Hydroxyle hervorgeht, und dass die dadurch frei werdenden Affinitäten der beiden Carbonyle sich gegenseitig sättigen:

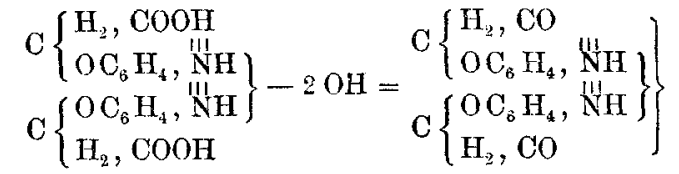

$$
\begin{aligned}
& \text { Hydrazoorthophenoxyl- } \quad \text { O-Amidophenoxylessig- } \\
& \text { essigsäure sàureanhydrid } \\
& \text { sog. ,inneres Anhydrid“. }
\end{aligned}
$$

Da der experimentelle Beweis der hier geäusserten Ansichten noch nicht beigebracht ist, so sind dieselben vor der Hand natürlich rein hypothetisch, und müssen die weiteren Untersuchungen über das O-Amidophenoxylessigsäureanhydrid und das Monochlororthoamidophenoxylessigsäureanhydrid über ihre Berechtigung entscheiden.

Die in Vorhergehenden beschriebenen Versuche haben folgende positive Resultate ergeben:

1) Die beste Methode zur Darstellung von O-Nitrophenoxylessigsäure ist die, äquivalente Mengen von O-Nitrophenolnatrium und monochloressigsaurem Natron in concentrirter wässriger Lösung 10-12 Stunden lang auf $100^{\circ} \mathrm{zu}$ erhitzen.

2) Durch Reduction der O-Nitrophenoxylessigsäure in alkalischer Lösung mittelst Natriumamalgam werden der Reihe nach Azoxyorthophenoxylessigsäure, Azoorthophenoxylessigsäure, Hydrazoorthophenoxylessigsäure und O-Amidophenoxylessigsäure - beziehentlich das Anhydrid der letzteren - erhalten.

$$
\mathrm{C}_{8} \mathrm{H}_{7} \mathrm{NO}_{5}
$$

O-Nitrophenoxylessigsäure

$$
\mathrm{C}_{16} \mathrm{H}_{14} \mathrm{~N}_{2} \mathrm{O}_{7}
$$

Azoxyorthophenoxylessigsäure

$$
\mathrm{C}_{19} \mathrm{H}_{1 !} \mathrm{N}_{z} \mathrm{O}_{6}
$$

Azoorthophenoxylessigsäure 
Blomstrand: Ein Uranmaterial von Moss etc. 191

$$
\begin{gathered}
\mathrm{C}_{16} \mathrm{H}_{16} \mathrm{~N}_{2} \mathrm{O}_{6} \\
\text { Hydrazoorthophenoxylessigsäure } \\
\mathrm{C}_{8} \mathrm{H}_{9} \mathrm{NO}_{3} \\
\text { O-Amidophenoxylessigsäure. }
\end{gathered}
$$

3) Durch Reduction der O-Nitrophenoxylessigsäure mit Eisenfeile und Essigsäure gelingt es zwar leicht, O-Amidophenoxylessigsäureanhydrid, aber nicht die von der O-Nitrophenoxylessigsäure sich ableitenden Azoverbindungen zu erhalten.

4) Durch Reduction der O-Nitrophenoxylessigsäure mit Zinnchlorür in salzsaurer Lösung entsteht in erster Linie Monochlororthoamidophenoxylessigsäureanhydrid, daneben bildet sich, namentlich bei unvollkommener Reduction, noch O-Amidophenoxylessigsäureanhydrid.

Leipzig, im Februar 1884.

\section{Ein Uranmineral von Moss und über die natür- lich vorkommenden Uranate im Allgemeinen; \\ von \\ C. W. Blomstrand. ${ }^{1}$ )}

Vor einigen Jahren hatte Prof. W. C. Brögger in den Feldspathgruben der Umgebung von Moss an der Ostküste des Christianiafjords eine reiche Fundgrube für verschiedene Mineralien entdeckt, welche überhaupt derselben Art sind, wie die für den scandinarischen Pegmatitgranit charalteristischen (Tantaloniobate, Phosphate u. s. w. der seltenen Frdarten). Ich war von Prof. Brögger gebeten worden, die Mineralien chemisch zu untersuchen, während er selbst das Studium der krystallographischen Merkmale unternahm. Die Arbeit wurde viel zeitraubender, als ich im Voraus erwartet hatte; andere chemische Fragen nahmen zeitweise meine ungetheilte

1) Von dem Hrn. Verf. nach Geol. Föreningars Förhandlingar Bd. 7, S. 59 (1884) bearbeitet. 